Research Article: New Research / Disorders of the Nervous System

\title{
Distinct aging-vulnerable and -resilient trajectories of specific motor circuit functions in oxidation- and temperature-stressed Drosophila
}

https://doi.org/10.1523/ENEURO.0443-21.2021

Cite as: eNeuro 2021; 10.1523/ENEURO.0443-21.2021

Received: 18 October 2021

Revised: 2 November 2021

Accepted: 5 November 2021

This Early Release article has been peer-reviewed and accepted, but has not been through the composition and copyediting processes. The final version may differ slightly in style or formatting and will contain links to any extended data.

Alerts: Sign up at www.eneuro.org/alerts to receive customized email alerts when the fully formatted version of this article is published. 4.0 International license, which permits unrestricted use, distribution and reproduction in any medium provided that the original work is properly attributed. 


\section{Title:}

Distinct aging-vulnerable and -resilient trajectories of specific motor circuit functions in oxidation- and temperature-stressed Drosophila

\section{Abbreviated Title:}

Aging-vulnerable and -resilient motor circuit properties

\section{Authors:}

Atulya Iyengar ${ }^{1,2,3, \dagger}$, Hongyu Ruan ${ }^{1, *, \dagger}$, and Chun-Fang $\mathrm{Wu}^{1,2,3}$

${ }^{1}$ Department of Biology, University of Iowa, Iowa City, IA 52242, USA. ${ }^{2}$ Iowa Neuroscience Institute, University of Iowa, Iowa City, IA 52242, USA. ${ }^{3}$ Interdisciplinary Graduate Program in Neuroscience, University of Iowa, Iowa City, IA 52242, USA.

*Present Affiliation: Department of Psychiatry, SUNY Upstate Medical University, Syracuse, NY 13210

${ }^{\dagger}$ Equal contributions

Author Contributions: AI, HYR, CFW designed research; AI, HYR performed research; AI, HYR, CFW analyzed data; AI, HYR, CFW wrote the paper.

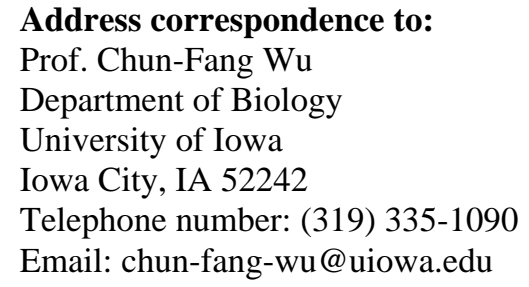

\section{Manuscript Information:}

Number of Figures: 7 (3 extended data figures); Number of Tables: 1; Number of Multimedia: 0; Abstract: 219 words; Significance statement: 120; Introduction: 612; Discussion: 3107 words

\section{Acknowledgements:}

We thank Xiaomin Xing, Scott Woods, Tristan O'Harrow and Atsushi Ueda for critical discussions during the projects, Jeff Nirschl, Jordan Imoehl, Kelle Goranson, and Amy Hoehne for assistance in data collection.

\section{Conflict of Interest:}

The authors declare that no conflict of interest exists.

\section{Funding Sources:}

This research was supported by a University of Iowa Biological Sciences Funding Program Grant and NIH grants, GM088804 AG047612, and AG051513 to CFW; and by an NIH PreDoctoral NRSA NS082001 and Iowa Neuroscience Institute Post-Doctoral Fellowships to AI 
Title:

2 Distinct aging-vulnerable and -resilient trajectories of specific motor circuit functions in

3 oxidation- and temperature-stressed Drosophila

5 Abstract

In Drosophila, molecular pathways affecting longevity have been extensively studied. However, corresponding neurophysiological changes underlying aging-related functional and behavioral deteriorations remain to be fully explored. We examined different motor circuits in Drosophila across the lifespan and uncovered distinctive age-resilient and agevulnerable trajectories in their established functional properties. In the giant-fiber (GF) and downstream circuit elements responsible for the jump-and-flight escape reflex, we observed relatively mild deterioration toward the end of lifespan. In contrast, more substantial agedependent modifications were seen in the plasticity of GF afferent processing, specifically in use-dependence and habituation properties. In addition, there were profound changes in different afferent circuits that drive flight motoneuron activities, including flight pattern generation and seizure spike discharges evoked by electroconvulsive stimulation. Importantly, in high temperature (HT)-reared flies $\left(29^{\circ} \mathrm{C}\right)$, the general trends in these age-dependent trajectories were largely maintained, albeit over a compressed time scale, lending support for the common practice of HT rearing for expediting Drosophila aging studies. We discovered that shortened lifespans in $\mathrm{Cu} / \mathrm{Zn}$ superoxide dismutase ( $\mathrm{Sod}$ ) mutant flies were accompanied by altered aging trajectories in motor circuit properties distinct from those in HT-reared flies, highlighting differential effects of oxidative vs temperature stressors. This work helps to identify several age-vulnerable neurophysiological parameters that may serve as quantitative indicators for assessing genetic and environmental influences on aging progression in Drosophila.

\section{Significance statement}

Comparisons of the aging trajectories of performance changes of several motor circuits in Drosophila revealed remarkably heterogeneous age-progressions. We identified "aging-resilient" and "aging-vulnerable" circuits in both normal control and flies with shortened lifespans due to either elevated rearing temperature or oxidative stress. Motor circuit components including flight motor neuron and the giant-fiber pathway responsible for the escape reflex showed only mild functional decline, whereas distinct trajectories throughout lifespan were seen in the flight 
33 pattern generator, interneuron inputs to the giant-fiber system, and circuits generating seizure

34 discharge patterns. Notably, high-temperature rearing generally compressed aging trajectories

35 while Sod mutation-induced oxidative stress led to distinct patterns of motor defects. Together,

36 these results elucidate potentially salient neurophysiological markers for aging in flies.

37

38

39

40

41 


\section{Introduction}

Aging nervous systems manifest a progressive functional decline. Invertebrate preparations offer experimentally tractable, simpler nervous systems with individually identifiable neurons to reveal the molecular and cellular bases of age-related neurophysiological changes (Atwood, 1992; Janse et al., 1986; Kenyon, 2001; Pletcher et al., 2002; Toivonen \& Partridge, 2009; Yeoman \& Faragher, 2001). In Drosophila, characteristic declines over the lifespan have been documented in motor coordination (Gargano et al., 2005; Leffelaar \& Grigliatti, 1983) and in higher functions, such as learning and memory (Tamura et al., 2003; Yamazaki et al., 2007). However, fewer studies have directly assessed cellular physiological decline underlying changes in behavioral performance across the lifespan (Banerjee et al., 2021; Martinez et al., 2007). It is important, therefore, to identify the neural circuits and associated behavioral outputs that are prone to age-related modifications and determine whether specific neuronal elements are differentially vulnerable in this process. Given that environmental and genetic contributions must be both considered in studies of the aging process (Curtsinger et al., 1995; Mair et al., 2003; Miquel et al., 1976), it is equally important to examine how ageprogression of circuit function is differentially modified by extrinsic environmental and intrinsic genetic factors. Such analyses may also uncover salient physiological parameters suitable for the assessment of aging progression.

This study examines several motor-pattern circuits in Drosophila whose outputs converge on a small subset of identified, well-described, flight motor neurons that innervate the indirect flight muscles (dorsal longitudinal muscles, DLM a-f, each receiving input from a single motor neuron, see Levine, 1973). Thus the DLM readout, driven by different motor circuits, enabled us to determine how age-dependent changes in different categories of motor performance are modified by either high-temperature rearing $\left(29^{\circ} \mathrm{C}\right.$, leading to a shortened lifespan), or oxidative stress in short-lived $\mathrm{Cu} / \mathrm{Zn}$ Superoxide dismutase (Sod) mutants (defective in a major free radical-scavenging enzyme, see Campbell et al., 1986; Phillips et al., 1989). Rearing flies at 29 ${ }^{\circ} \mathrm{C}$ is a widely adopted protocol for experimental expedience in Drosophila aging and neurodegeneration studies (e.g. Biteau et al., 2010; Kang et al., 2002; Min \& Benzer, 1997; Simon et al., 2003; Sudmeier et al., 2015). Our study directly investigated the impact of rearing at $29{ }^{\circ} \mathrm{C}$ on different motor functions over the lifespan, as well as the cellular physiological links between proposed oxidative stress mechanisms (Finkel \& Holbrook, 2000; Harman, 1956; 
Ziegler et al., 2015) and the associated behavioral alterations previously described for Sod mutant flies.

We also present an overall assessment of the trajectory of neural circuit performance associated with different categories of motor behaviors along normal and altered lifespans. These include flight pattern generation (Harcombe \& Wyman, 1977; Levine, 1973) the giant fiber (GF) pathway-mediated jump and flight escape reflex (Engel \& Wu, 1992; Gorczyca \& Hall, 1984; Tanouye \& Wyman, 1980), habituation of the GF pathway escape response to repetitive stimulation (a form of non-associative learning, Engel \& Wu, 1996; Engel et al., 2000) and a stereotypic seizure repertoire triggered by electroconvulsive stimulation (ECS, Kuebler \& Tanouye, 2000; Lee et al., 2019; Lee \& Wu, 2002, 2006; Pavlidis \& Tanouye, 1995). Our analysis has identified age-resilient and age-sensitive circuit properties that are attributable to changes in distinct circuit components. Furthermore, we found WT flies reared at $29{ }^{\circ} \mathrm{C}$ manifested accelerated trajectories of age-dependent alterations resembling those at $25^{\circ} \mathrm{C}$ at a normalized timescale, while Sod mutants displayed distinctively altered pattern of aging progression. Our findings also identify several neurophysiological parameters that could serve as quantitative indices for assessing age-related functional decline. These findings have been reported in part in PhD dissertations (Iyengar, 2016; Ruan, 2008) and in abstract form (Iyengar et al., 2010; Ruan \& Wu, 2009; Wu \& Ruan, 2008).

\section{Methods}

\section{Fly Stock Maintenance and Lifespan Assays}

The Canton-S wild-type (WT) strain and Sod mutant allele $\operatorname{Sod}^{l}$ were maintained on standard cornmeal medium (Frankel \& Brousseau, 1968). Originally isolated in an EMS mutagenesis screen as $l-108$ (Campbell et al., 1986), the $\operatorname{Sod}^{l}$ allele (also known as $\operatorname{Sod}^{n 108}$ ) was recombined on a red chromosome background (Phillips et al., 1989), and kept as a balanced stock $\operatorname{Sod}^{1}$ red/ TM3, Sb Ser. Among Sod alleles previously studied (e.g. Sod ${ }^{x 39}, \operatorname{Sod}^{n 58}, \operatorname{Sod}^{n 64}$ all on the red background, $\operatorname{Sod}^{21}$ on a white background), $\operatorname{Sod}^{1}$ was selected for this study because this allele has been well characterized and could be more readily managed to generated sufficient numbers of flies for cross-sectional comparisons across the lifespan (Ruan \& Wu, 2008; Şahin et al., 2017). For collecting rare homozygous offspring of Sod flies, the stock was grown in half-pint bottles to encourage reproduction. Newly eclosed adults were collected under 
$\mathrm{CO}_{2}$ anesthesia (WT every 1-2 days, Sod flies every 2 days) to assemble sufficient sample sizes to initiate lifespan assays. Flies were distributed into food vials (10-14 flies per vial for WT, 7 10 flies for $S o d$ ) and were transferred to fresh vials every 2 days. All vials were pre-warmed to the corresponding temperature before fly transfers. Foam plugs were used and the vials were kept horizontally to avoid weaker flies being accidentally stuck to food or cotton plugs. Flies were maintained in incubators set at $25{ }^{\circ} \mathrm{C}$ and $29{ }^{\circ} \mathrm{C}$ or $23{ }^{\circ} \mathrm{C}$, were kept at the ambient humidity range of $\sim 30-70 \%$, and under 12:12 h light:dark conditions. Survivors were counted and dead flies were removed daily. When reported as a function of $\%$ mortality, flies were sampled within one day of the age corresponding with \% mortality (see Results and Figure 1A).

\section{Flight Assessment and Indirect Flight Muscle Recording}

Preparation, stimulation, recording, and analysis of dorsal longitudinal muscle (DLM) responses have been described previously (Engel \& Wu, 1992; Iyengar \& Wu, 2014; Lee \& Wu, 2002). Flies were anesthetized briefly on ice or by light ether exposure and glued to a metal wire between the head and thorax using a nitrocellulose or cyanoacrylate based adhesive (Gorczyca \& Hall, 1984). Mounted flies were allowed at least $30 \mathrm{~min}$ rest in a humid chamber before recording. All electrophysiological and behavioral experiments were carried out at room temperature $\left(21-23^{\circ} \mathrm{C}\right)$.

Flights were triggered via a gentle $500 \mathrm{~ms}$ air puff generated by an aquarium air pump (Whisper 10-30 Tetra, Blacksburg, VA, USA) pumping air through a $4 \mathrm{~mm}$ diameter tube placed approximately $4 \mathrm{~mm}$ away from the fly. A 3-way solenoid valve (ASCO scientific, P/N AL4312, Florham Park, NJ, USA) driven by a USB 6210 DAQ card (National Instruments, Austin TX) controlled air flow. Wing sounds were picked up by a high-gain microphone placed approximately $4 \mathrm{~mm}$ below the fly. Acoustic signals were digitized by a PC sound card controlled by a LabVIEW 8.6 script. The wing beat frequency was determined using the shorttime FFT procedure described in Iyengar \& Wu (2014).

Action potentials from the top-most dorsal longitudinal muscle (\#45a, Miller, 1950) were recorded by an electrolytically sharpened tungsten electrode penetrating the flight muscle with a similarly constructed electrode inserted in the abdomen for reference. Signals were accessed with an AC amplifier (Microelectrode AC Amplifier, Model 1800, A-M Systems, Inc., Carlsborg, WA; filter bandwidth from $10 \mathrm{~Hz}$ to $20 \mathrm{kHz}$ ). Amplified signals were recorded with pulse code 
135

modulation (Neuro-Corder DR-484, Cygnus Technology, Inc., Delaware Water Gap, PA) on videotape at a sampling rate of $44 \mathrm{kHz}$. Digitization of spiking traces (Figures 1, 6 and Figure 51) was carried out by a digital acquisition card (USB 6210) controlled by custom-written LabVIEW scripts.

\section{Giant Fiber Pathway Stimulation}

As previously described (Engel \& Wu, 1996), a pair of uninsulated tungsten electrodes were inserted in the eyes (anode in left eye) to pass stimulation of $0.1 \mathrm{~ms}$ duration (Isolated Pulse Stimulator, Model 2100, A-M Systems, Inc., Carlsborg, WA). Long-latency and short-latency response (LLR and SLR) thresholds were first determined using increasing stimulation intensity with an inter-stimulus interval of $30 \mathrm{~s}$. For the experiments involving LLR (twin-pulse refractory period and habituation), stimulus intensity was set at least $0.5 \mathrm{~V}$ below the SLR threshold. For SLR-related trials, stimulus intensity was set at $20 \mathrm{~V}$, which was well above the SLR thresholds in all flies tested (see Engel and Wu, 1992 for details).

To determine the refractory period of LLR and SLR, twin-pulse stimuli were given every $10 \mathrm{~s}$, with the stimulus interval decreasing stepwise (step size: 5 - $10 \mathrm{~ms}$ for LLR, $0.5-1 \mathrm{~ms}$ for SLR) from a starting interval (100 - $800 \mathrm{~ms}$ for LLR, 10 - $20 \mathrm{~ms}$ for SLR). When a given interstimulus interval failed to trigger a second DLM spike in one or two trials, a 3 - 5 min rest was allowed before a train of six twin-pulses of the same inter-stimulus interval were delivered at 0.1 $\mathrm{Hz}$ between twin pulses. If all six trials failed, then the previous stimulus interval was recorded as the refractory period.

The ability of GF to follow high-frequency stimulation was determined as described previously (Engel \& Wu, 1992; Gorczyca \& Hall, 1984). Three trains of 10 pulses at $200 \mathrm{~Hz}$ with $10 \mathrm{~s}$ in between were delivered and the number of responses was counted.

\section{Habituation of GF-Mediated LLRs and Electroconvlusive Stimulation-Evoked Seizures}

For testing habituation rate, two trials of 100-pulse LLR stimulation trains at a given frequency $(1,2$, and $5 \mathrm{~Hz})$ were delivered with a $10 \mathrm{~min}$ interval between trials. The criterion for reaching habituation was the occurrence of the first five consecutive LLR failures (F5F, Engel \& $\mathrm{Wu}, 1996)$. The larger F5F value of the two trials was recorded for the fly tested. Upon 
reaching habituation criterion, an air puff dishabituation stimulus was delivered to confirm habituation (see Engel and Wu, 1996 for additional details).

High-frequency electroconvulsive stimulation $(0.1-\mathrm{msec}$ pulses at $200 \mathrm{~Hz}$ for $2 \mathrm{~s}$ at a particular voltage $15-80 \mathrm{~V}$ ) were delivered across the brain to induce a stereotypic electroconvulsive seizure (ECS) discharge pattern (see Lee and Wu, 2002 for details). After high-frequency stimulation, test pulses of 20 volts or higher were sometimes delivered at $1 \mathrm{~Hz}$ to examine the failure and recovery of the GF pathway motor response. An interval of at least 10 min was allowed between each ECS to avoid the effect of refractoriness (Lee \& Wu, 2002). For follow-up experiments of DLM spike patterning during ECS discharges (Figure 6 and Figure 5-1), spikes were detected and their timing recorded using custom-written Matlab scripts (Iyengar \& Wu, 2014; Lee et al., 2019). Firing rate was measured by the instantaneous firing frequency $\left(\right.$ ISI $^{-1}$, defined as the reciprocal of the inter-spike interval, ISI between successive spikes), and firing regularity was quantified by the instantaneous coefficient of variation $\left(\mathrm{CV}_{2}\right.$ see Holt et al., 1996; Lee et al., 2019). Poincaré trajectories were constructed by plotting the ISI $^{-1}$ of a spike interval against the $\mathrm{ISI}^{-1}$ of the subsequent interval. Averaged $\mathrm{ISI}^{-1}$ vs $\mathrm{CV}_{2}$ plots were constructed as described in Lee et al. (2019).

The neurophysiological properties of each fly were tested in the following sequence with 5 min rest in-between to minimize interference between protocols: flight, LLR and SLR thresholds, LLR refractory period, LLR habituation, SLR refractory period, ability of SLR to follow high frequency stimulation (30 pulses at $200 \mathrm{~Hz}$ ), and ECS-evoked seizure discharges. Due to the weakness of aged flies and in some cases the absence of LLRs in the last 5\% survivors, not all of the flies went through every protocol. If the LLR was absent, all SLR-related protocols were still examined. Generally, all electrophysiological protocols took less than one hour to complete for a single fly.

\section{Statistical Analysis}

For each age group tested (1, 5, 50 and 95\% mortality), between 7 and 12 flies were generally tested. We have found that this sample size range is often sufficient to draw initial conclusions, based on our previous experiences studying ion channel and $2^{\text {nd }}$ messenger system mutants (Engel \& Wu, 1992, 1996; Iyengar \& Wu, 2014; Lee \& Wu, 2006). Individual flies were considered biological replicates, and all recorded data points were included in the analysis. Due 
to the non-normal distribution of datasets, the non-parametric Kruskal-Wallis ANOVA test was used to determine cross-sectional differences between physiological parameters of flies from respective genotypes. The rank-sum test (with Bonferroni correction applied) was used as a post hoc test. Age-dependent trends in physiological parameters were identified by computing the non-parametric Spearman's rank correlation coefficient $\left(\mathrm{r}_{\mathrm{s}}\right)$ (Sokal \& Rohlf, 1969). In the source data files accompanying each figure, the sample mean, median, standard deviation, coefficient of variation and the $25-75 \%$ tile interval are listed. To assess the robustness of the respective statistics, a bootstrap resampling approach (Efron, 1981) with 1000 replicates was used to estimate the $95 \%$ tile confidence intervals. All statistical analyses were performed in Matlab (r2019b).

\section{Results}

Flight Performance and DLM Firing Activity across the Lifespan: Effects of High-Temperature Rearing and Sod Mutation.

The remarkable flight ability of Drosophila has been well documented (Dickinson, 2014; Heisenberg \& Wolf, 1984), with flights often lasting for hours in tethered flies (Götz, 1968; Götz, 1987). To assess age-related changes in flight ability over the lifespan, we examined sustained tethered flight (Figure 1A, cf. Iyengar \& Wu, 2014) to delineate characterisic alterations in flight muscle electrical activity patterns and corresponding biomechanical parameters, and to reveal the effects of high-temperature and oxidative stressors on the aging process.

As established in Drosophila and other organisms, the aging process manifests a nonlinear progression, indicated by varying rates of mortality at different stages in the lifespan (Figure 1B, cf. Vaupel et al., 1998, see Extended Data Figure 1-1 for the log-transformed mortality rate). We examined WT flies at 25 and $29^{\circ} \mathrm{C}$; two commonly used rearing temperatures in Drosophila aging studies. As previously reported, at $29^{\circ} \mathrm{C}$ the fly lifespan was nearly halved as compared to $25^{\circ} \mathrm{C}$ with a substantially accelerated mortality rate, convenient features frequently used to expedite aging studies (Figure 1B; Leffelaar \& Grigliatti, 1983; Loeb \& Northrop, 1917; Ruan \& Wu, 2008). At both temperatures, however the lifespan trajectories follow an inverse sigmoidal curve, with "plateau", "shoulder" and "tail" phases (Curtsinger et al., 1992). We have also determined the effects of increased oxidative stress by characterizing 
227

Sod mutant flies, reared at a slightly lowered rearing temperature, $23{ }^{\circ} \mathrm{C}$ rather than $25{ }^{\circ} \mathrm{C}$ in order to expand the rather short Sod lifespan for more precise chronological tracking of data. Notably, in the $\operatorname{Sod}$ lifespan curve at $23{ }^{\circ} \mathrm{C}$, the early plateau phase is absent (Figure 1B), and the slope of the mortality rate is negative across the lifespan (Extended Data Figure 1-1). These distinctive features are previously reported Sod life-span curves obtained at higher temperatures ( 25 or $29^{\circ} \mathrm{C}$, Phillips et al., 1989; Ruan \& Wu, 2008), and highlight the characteristic high mortality of young Sod flies.

We found that flight ability was well-preserved in some aged WT flies reared at either 25 or $29^{\circ} \mathrm{C}$, displaying robust tethered flights beyond the 30 -s sustained flight duration, a cut-off criterion which effectively distinguishes flight-defective mutants from WT individuals (Iyengar $\& \mathrm{Wu}, 2014)$. The "non-fliers" were mostly encountered in flies aged beyond the median lifespan (55 and $30 \mathrm{~d}$ for individuals reared at 25 and $29{ }^{\circ} \mathrm{C}$ respectively, Figure $1 \mathrm{C}$ ). In striking contrast, many young Sod flies ( $\sim 50 \%)$ were not capable of sustained flight beyond $30 \mathrm{~s}$, and this proportion further increased with age, with few Sod flies beyond the median lifespan ( $9 \mathrm{~d}$ ) capable of flight beyond $30 \mathrm{~s}$ (Figure 1C).

The differences between WT and Sod mutants in flight ability prompted us to examine the wing beat frequency (WBF) as well as flight muscle DLM firing rate, across the lifespan. Using protocols established for the tethered flight preparation (Iyengar \& Wu, 2014), we were able to acoustically monitor WBF via a microphone and simultaneously record DLM spiking activity (Figure 1A, D). The WBF is largely determined by the natural mechanical resonance frequency of the thorax case, which is powered by alternating activation of two sets of indirect flight muscles, DLMs and dorsal-ventral muscles or DVMs (Chan \& Dickinson, 1996). Isometric contraction of indirect flight muscles is stretch-activated and coincides with mechanical oscillation of the thorax, while their spiking activity, occurring at a much lower frequency than WBF, facilitates $\mathrm{Ca}^{2+}$ influx for force generation (Dickinson \& Tu, 1997; Lehmann \& Dickinson, 1997). Therefore, changes in muscle tension without shortening allow microelectrode recordings of the DLM spikes with minimal cell damage during prolonged flight.

Across the lifespan of WT flies, we noted a clear trend of increasing DLM firing rates which nearly doubled in the oldest flies examined (Figure 1D-E, Spearman rank correlation coefficient: $r_{s}=0.46, p=9.9 \times 10^{-4}$, and $r_{s}=0.58, p=3.6 \times 10^{-4}$, respectively, for 25 and $29^{\circ} \mathrm{C}$ reared flies). Accompanying this increasing trend, we noted an apparent overall increase in 
variability (Figure 1E, dashed distribution outline). In contrast to this age-associated variability in DLM firing, the WBF showed little change throughout lifespan for the fliers in the three populations. In $25^{\circ} \mathrm{C}$-reared WT flies both young and old individuals displayed average WBF of $\sim 190 \mathrm{~Hz}$, with a narrower spread within a $+/-20 \mathrm{~Hz}$ (Figure 1F). We observed only a small, but detectable, reduction in frequency across the lifespan of $29^{\circ} \mathrm{C}$ reared flies $\left(\mathrm{r}_{\mathrm{s}}=-0.41, \mathrm{p}=\right.$ 0.19). Remarkably, the small population of Sod fliers displayed largely normal DLM firing rate and $\mathrm{WBF}$ with an indicative upward trend in both DLM firing rate and $\mathrm{WBF}\left(\mathrm{r}_{\mathrm{s}}=0.50, \mathrm{p}=0.06\right.$ and $r_{s}=0.60, p=0.018$, respectively, Figure 1E-F). Taken together, our analysis of flight patterns suggests that the biomechanical properties of flight remained largely stable across the lifespan, while increases in DLM spiking mean frequency and variability reflected potential agerelated alterations in central motor circuits.

In the following sections, we adopt the measure of "biological age", by tracking the percent mortality of the population, in order to facilitate comparisons of the aging trajectories of functional alterations across different genotypes and conditions. This can be achieved by a renormalization of the time axis to reflect the cumulative mortality based on the chronology lifespan curves (Figure 1B). As shown in the following figures, we collected data at different biological ages, for WT flies reared at $25{ }^{\circ} \mathrm{C}$, biological ages at $<1 \%, 5 \%, 50 \%$ and $95 \%$ mortality corresponded to the chronological ages of 7, 31, 50 and 72 days, whereas for $29^{\circ} \mathrm{C}$ rearing, this shifted to 7, 20, 30 and 35 days. Since Sod flies lacked a prolonged initial plateau phase but showed a lingering "tail" in the lifespan curve, the biological ages targeted for data collection were modified to compress the first time interval to $<5 \%$ mortality, and $70 \%$ mortality was added before the final $95 \%$ stage so as to allow sampling within the prolonged "tail". The stages for Sod flies at <5\%,50\%,70\%, and 95\% mortality therefore corresponded to 2, 9, 14, and 30 days. The distinct profile of Sod aging trajectory indicates markedly different effects exerted by oxidative and high temperature stressors on the mortality rate along the lifespan. It is worth noting that despite the early precipitous drop and a shortened population medium lifespan, a small fraction of Sod mutant flies nevertheless exhibited relatively prolonged survivorship, giving rise to the characteristic, disproportionally prolonged tail in its lifespan curve.

Age-Resilient and-Vulnerable Properties of the Giant Fiber (GF) Circuit that Mediates a Jumpand-Flight Escape Reflex 
One of the best-studied motor circuits in adult Drosophila is the giant fiber (GF) pathway that triggers a jump-and-flight escape reflex critical for survival (Figure 2A, cf. Tanouye \& Wyman, 1980; Trimarchi \& Schneiderman, 1995b; von Reyn et al., 2017). Visual and other sensory inputs to the GF interneuron dendrites in the brain initiate the action potential propagating along the descending axon to drive a set of motor neurons in the thoracic ganglion. The DLM motor neuron receives the GF command signal indirectly via a peripherally synapsing interneuron (PSI) to evoke a DLM spike. This axo-axonal GF-PSI contact has been characterized as a mixed electrical and cholinergic synapse (Allen \& Murphey, 2007; Blagburn et al., 1999; Gorczyca \& Hall, 1984; King \& Wyman, 1980). It has been well established that electric stimuli across the brain evoke two types of GF pathway-mediated DLM responses with distinct latencies (Figure 2B). Low-intensity stimulation across the brain recruits afferent inputs to the GF neuron, thereby triggering a relatively longer latency response (LLR, 4 - 6 ms, Elkins \& Ganetzky, 1990; Engel \& Wu, 1992, 1996), whereas high-intensity stimuli directly activate GF axonal spikes bypassing the brain afferents and resulting in a shorter latency response (SLR, $\sim 0.8$ - 1.6 ms, Engel \& Wu, 1992; Tanouye \& Wyman, 1980).

We found clear distinctions between SLR and LLR in age-dependent modifications across the lifespan. Figure 2C shows robust maintenance of the basic properties (axonal conduction), of the GF neuron and downstream elements (PSI and DLM motor neurons) in aging WT flies, as indicated by the well preserved SLR parameters (see Table 1 for sample means, standard deviations and coefficients of variation). No significant age-dependent alterations were observed in the latency or threshold of the SLR for either 25 or $29^{\circ} \mathrm{C}$-reared WT flies, consistent with a previous report on well-maintained SLR properties up to a late stage of the lifespan (cf. Martinez et al., 2007). Nevertheless, we did observe a significant change in SLR properties in young Sod mutants which displayed a retarded latency compared to $25^{\circ} \mathrm{C}$-reared WT counterparts (SLR latency mean: 1.6 vs $1.1 \mathrm{~ms}, \mathrm{p}=0.002$, Kruskal-Wallis ANOVA, Figure 2C, Table 1). Furthermore, we noted an age-dependent decrease in this parameter for $\operatorname{Sod}\left(\mathrm{r}_{\mathrm{s}}=-0.50\right.$, $\mathrm{p}=0.0015)$. This apparently paradoxical improvement brought the SLR latency parameters to a range directly comparable to those of WT flies, likely reflecting a progressive change in the Sod population composition, as healthier individuals persist and become better represented, while weaker ones die off. 
In contrast to the robust SLR properties, LLR parameters revealed clear changes in afferent inputs from higher centers to the GF neuron. Strikingly, among the oldest WT flies (e.g. $25^{\circ} \mathrm{C}, 95 \%$ mortality), LLRs were not elicited in more than half of them (14 of 26), indicating severely compromised afferent inputs to the GF. Additionally, both 25 and $29{ }^{\circ} \mathrm{C}$-reared WT populations displayed a small $(<10 \%)$, but detectable, age-dependent increases in LLR latency $\left(r_{s}=0.37\right.$ and $0.47 ; p=0.003$ and 0.0005 , respectively, see also Table 1$)$, and a modest agedependent increase in LLR threshold $\left(r_{s}=0.57, p=1.3 \times 10^{-5}\right)$ was found in $29^{\circ} \mathrm{C}$-reared flies. These relatively stable LLR properties were not grossly disrupted in the Sod mutant flies that remained responsive (Figure 2D).

In summary, our SLR results indicate that the GF and its downstream elements, including the PSI and DLM motor neurons remain robust and could reliably transmit vital signals to evoke an escape reflex even in aged flies. However, the input elements upstream to the GF neuron showed more clear age-dependent functional decline, which could lead to a catastrophic collapse of LLR in some of the extremely old individuals (95\% mortality) that were devoid of LLR.

\section{Alteration of SLR and LLR Refractory Period by High-Temperature and Oxidative Stressors}

The robust, well-maintained basic properties of the GF and its downstream elements provide reliable readouts for examination of activity-dependent plasticity across the lifespan to uncover aging-vulnerable neural elements upstream of the GF neuron. By employing wellestablished stimulus protocols in the tethered fly preparation for activity-dependent processes, including paired-pulse refractory period, and habituation to repetitive stimulation (Engel \& $\mathrm{Wu}$, 1996; Gorczyca \& Hall, 1984), we were able to establish modification due to aging in these usedependent properties, as well as their alterations by high-temperature or oxidative stressors. The refractory period (RP), for both SLR and LLRs, is defined as the shortest interval between paired stimulus pulses at which a second DLM response could be evoked (Figure 3A inset). We found WT flies across the lifespan displayed similar SLR RP performance when reared at $25^{\circ} \mathrm{C}$, while $29^{\circ} \mathrm{C}$-reared WT flies displayed an apparent, but not statistically significant trend of increasing RP over the lifespan. In a complimentary set of experiments, we delivered 30 SLRevoking stimuli at $200 \mathrm{~Hz}$ and recorded the number successful responses $\left(\mathrm{FF}_{30}\right)$. Across the lifespan, both $25^{\circ} \mathrm{C}$ and $29^{\circ} \mathrm{C}$-reared flies displayed small alterations in performance, with changes not reaching statistical significance (Extended Data Figure 3-1). The refractoriness of 
LLR in older WT flies reared at $25^{\circ} \mathrm{C}$ was similar their younger counterparts. However, $29{ }^{\circ} \mathrm{C}$ reared WT flies showed a significant age-dependent increase in LLR RP, most evident in the older populations (Figure 3B, 50 \& 95\% mortality). Notably, the distributions of SLR and LLR RPs in aged groups showed larger skews with several individuals displaying disproportionally lengthened refractory periods (less pronounced in SLR but most evident in LLR RP, Figure 3B). Furthermore, the oldest groups (95\% mortality) consistently displayed the largest coefficients of variation of the LLR refractory periods datasets (Table 1). These observations highlight the stochastic nature of age-related functional deterioration in the upstream circuit elements afferent to the GF pathway.

Remarkably, young Sod flies represented the most heterogeneous population among different age groups in terms of refractory periods. Presumably the population included a portion of severely defective, very short-lived individuals that accounted for the initial drop and the diminished plateau phase of the lifespan curve (Figure 1B). Our observations revealed significant lengthening in both SLR and LLR refractory periods in very young ( $<5 \%$ mortality) Sod mutants (SLRs $\mathrm{p}<0.05$, and LLRs, $\mathrm{p}<0.01$, Figure 3A-B, Kruskal Wallis ANOVA, Bonferroni-corrected rank-sum post hoc test). Similarly, Sod mutants displayed significantly lower $\mathrm{FF}_{30}$. indicating worse performance compared to WT counterparts (Extended Data Figure 3-1). In conjunction with a clear increase in SLR latency (Figure 2C), the RP lengthening point towards severe GF defects in a sub-population of young Sod flies. On the other hand, the surviving aged Sod mutants showed relatively stable SLR and LLR responses, with less substantial age-dependence beyond 50\% mortality (Figure 3A-B).

\section{Aging Progression of Higher-Level Activity-Dependent Signal Processing: Acceleration of LLR}

\section{Habituation over Lifespan}

The above results suggest the functional integrity of the GF and its downstream elements as indicated by SLR performance (Figures $2 \& 3$ ) is age-resilient across the lifespan.

Nevertheless, high-temperature stress and particularly Sod mutation can exert clear effects on activity-dependent aspects of GF-mediated DLM responses, as indicated by refractory period of LLRs (Figure 3B). In Drosophila, the jump-and-flight startle reflex shows habituation upon repetitive visual stimulation (Trimarchi \& Schneiderman, 1995a), which is recapitulated in the habituation of LLRs (Engel \& Wu, 1996). Habituation represents a non-associative form of 
381 learning that involves experience-dependent plasticity in higher functions (Engel \& $\mathrm{Wu}, 2009$;

382 Rankin et al., 2009).

383 To further investigate age-related changes in the plasticity of upstream processing of GF

384 inputs, we examined habituation of LLRs (Engel \& Wu, 1996; 1998). Upon evoking LLRs

385 repetitively, the DLM responses habituate, as subsequent stimuli fail to recruit LLRs (Figure

386 4A). However, LLRs may be rapidly restored following the application of a dis-habituation

387 stimulus of a different modality, such as an air-puff, excluding sensory adaptation or motor

388 fatigue as the basis for LLR failure (Figure 4A). We applied 100 LLR-evoking stimuli at three

389 frequencies $(1,2$ and $5 \mathrm{~Hz}$ ), and adopted a habituation criterion as the number of stimuli required

390 to reach 5 consecutive LLR failures (F5F, Figure 4A-B, cf. Engel \& Wu 1996). The large

391 number of stimuli used in this protocol provides greater power to resolve age-dependent

392 alterations in an intrinsically stochastic process.

393 Across the three stimulation frequencies examined (1, 2 and $5 \mathrm{~Hz}$ ) for WT flies reared at

$39425{ }^{\circ} \mathrm{C}$, we found that between $<1 \%$ and $5 \%$ mortality, during the plateau portion of the lifespan,

395 the LLR habituation rate was largely unaltered with a large fraction of flies showing little

396 habituation throughout the stimulation train (Figure 4C-E). However, during the mortality phase

397 of the lifespan curve, $50 \%$ mortality and beyond, we observed a marked acceleration in LLR

398 habituation, with the oldest flies habituating the fastest ( $r_{s}$ between -0.33 to $-0.56, p<0.001$ ). As

399 expected, we found more evident acceleration in habituation rate at higher stimulation

400 frequencies (Figure 4C-E), which was most pronounced in the older populations (50 \& $95 \%$

401 mortality) examined. Together, our findings suggest that the brain circuits afferent to the GF

402 pathway remained largely unaltered during the plateau portion of the lifespan curve, a substantial

403 fraction of the lifespan. Nevertheless, across the lifespan, we could delineate the distinct

404 habituation rates associated with the 5\%,50\% and 95\% age groups, particularly at the higher

405 simulation frequency of $5 \mathrm{~Hz}$, offering a quantifiable index of aging progression.

$406 \quad$ High temperature rearing and Sod mutations further accelerated habituation. Compared to

$40725{ }^{\circ} \mathrm{C}$-reared counterparts, $29^{\circ} \mathrm{C}$-reared WT flies displayed considerably faster habituation to 1

$408 \mathrm{~Hz}$ stimulation in aged groups (50 and $95 \%$ mortality, Figure 4E vs. F). Furthermore, Sod

409 mutants (at $23{ }^{\circ} \mathrm{C}$ ) showed an accelerated habituation rates compared to WT counterparts even in

410 younger flies (<5\% mortality, Figure 4G vs, E \& F), presumably correlated with the pronounced

411 increase in LLR paired pulse refractory period in this age group (Figure 3B). Therefore, among 
412 the temperature- or oxidation-stressed groups, the habituation rate again can serve as a

413 physiological marker of age progression even at low frequencies $(\sim 1 \mathrm{~Hz})$ of repetitive

414 stimulation.

415

\section{Increasing Susceptibility to Electroconvulsive Induction of Seizures across the Lifespan}

In addition to acting as an output of the flight pattern generator and GF pathwaymediated escape reflex described above, DLM action potentials may serve as reliable indicators of CNS spike discharges associated with seizures (Lee \& Wu, 2002; Pavlidis \& Tanouye, 1995). Such seizure episodes may reflect aberrant spiking originating from motor pattern generators located in the thorax, such as flight (Harcombe \& Wyman, 1977; Iyengar \& Wu, 2014) and grooming (Engel \& Wu, 1992; Lee et al., 2019). As shown in Figure 5A, high-frequency stimulation across the brain $(200-\mathrm{Hz}, 2-\mathrm{s}$ train of $0.1-\mathrm{ms}$ pulses, Lee $\& \mathrm{Wu}, 2002)$ can trigger a highly stereotypic electroconvulsive seizure (ECS) discharge repertoire, consisting of an initial discharge (ID), a period of paralysis reflected by GF pathway failure (F, in response to $1-\mathrm{Hz}$ test pulses), and a second delayed discharge (DD) of spikes. This experimental protocol provides several stable and quantitative measures, within individual flies, suitable for assessing agerelated modifications of CNS function, including the threshold to induce seizures (by adjusting intensity from 15 to $80 \mathrm{~V}$, Figure 5B) and the discharge duration of DD spike trains (Figure 5C). We found a clear trend of increasing seizure susceptibility with age in WT and Sod flies (Figure 5B). WT flies reared either at 25 or $29^{\circ} \mathrm{C}$ displayed a substantial reduction in the DD threshold across their lifespan (-33\% and $-23 \%$ respectively for $<1 \%$ vs. $95 \%$ mortality). In Sod mutants, the trend was even more pronounced (-46\% for $5 \%$ vs. $95 \%$ mortality). The effects of the Sod mutation were most striking in the youngest age group. In these flies ( $<5 \%$ mortality), threshold was significantly higher than WT counterparts $(\mathrm{p}<0.001$, Kruskal-Wallis ANOVA, rank-sum post hoc), corroborating the other observations of extreme physiological defects found in this age group (Figures $2 \& 3$ ). Furthermore, this increase in ECS threshold was present across the Sod lifespan ( $\mathrm{p}<0.05$, Kruskal-Wallis ANOVA, rank-sum post hoc) suggesting that elevated oxidative stress could render seizure-prone circuits less excitable.

Despite individual variability within each fly group, significant correlation coefficients suggest an enhanced excitability of seizure-prone circuits in aged flies (Figure 5B). Compared with other potential indicators, e.g. habituation rate (Figure 4), this age-dependent monotonic 
decrease of ECS threshold could provide as a tighter, more quantitative measure of aging progression.

In contrast to the decreasing trend of seizure threshold over lifespan, there was a milder trend of increasing DD duration in WT flies reared at $29^{\circ} \mathrm{C}$, or in $\operatorname{Sod}$ mutants, but ending with a substantial drop in the oldest (95\%) populations (Figure 5C, $\mathrm{r}_{\mathrm{s}}=0.36, \mathrm{p}=0.0041$; and 0.34, $\mathrm{p}=$ 0.047 for WT $29^{\circ} \mathrm{C}$ and Sod respectively). However, there was no clear age-dependent trend of increasing DD duration in $25^{\circ} \mathrm{C}$-reared WT flies (Figure 5C). In a complementary data set acquired at higher temporal resolution to resolve individual spikes, we examined the total number of spikes during the DD in these populations. Mirroring the DD duration observations, we found that $25^{\circ} \mathrm{C}$-reared WT flies did not display an age-dependent trend, while WT flies reared at $29^{\circ} \mathrm{C}$, as well as $\operatorname{Sod}$ mutants, showed modest increases in the DD spike count (Extended Figure 5-1, $\mathrm{r}_{\mathrm{s}}=0.50, \mathrm{p}=1.4 \times 10^{-5}$ for WT $29^{\circ} \mathrm{C} ; \mathrm{r}_{\mathrm{s}}=0.27, \mathrm{p}=0.04$ for $\operatorname{Sod}$ mutants).

\section{Age-Trajectories of ID Firing Patterns Reveal a Potential Predictor of Mortality Rate}

Alterations in ECS threshold and DD duration across life span (Figure 5) call for detailed characterization of ID spiking to extract additional salient features associated with aging progression. Since ID occurs as a brief burst ( $1-4 \mathrm{~s}$, see Figures 5A and 6A) of high-frequency spiking activity, a set of ECS data at a higher temporal resolution enabled an analysis of ID spiking dynamics at the level of individual spikes (Figure 6A). Immediately following electroconvulsive stimulation, the ID spike patterns manifested striking, but more complex, age dependence and vulnerability to oxidative stress. In younger WT flies (reared at either 25 or 29

${ }^{\circ} \mathrm{C}$ ), ID spike counts progressively increased with age until about 50\% mortality (Figure 6A and B). Beyond 50\% mortality, a decline in the ID spike count became apparent; notably the oldest flies (95\% mortality) regressed to a spike count similar to those of young flies ( $<1 \%$ mortality). In contrast to nearly 10-fold range for ID spike counts across the WT lifespan, Sod mutants displayed only rather sparse spiking, too few to shape a prominent age-related profile in their counts (Figure 6A and B). This observation mirrors the particular vulnerability of ECS discharges to oxidative stress, along with the drastically elevated threshold levels in ECS induction in Sod mutant flies (Figure 5B).

We noted that the curve of average ID spike count across the lifespan showed a gross resemblance in overall profile with the mortality rate curve. The progression of spike count 
changes apparently preceded the mortality rate changes in WT (compare Figure 6B and C). We sought an appropriate temporal shift between the ID spike count and mortality rate curves that would optimize regression fit. The iterations yielded the best correlation between the ID spike count and mortality rate with 11- and 6-d lags for WT flies reared at 25 and $29{ }^{\circ} \mathrm{C}$, respectively (Figure 6D and E). These observations suggest that the ID spike production over the lifespan may serve a predictor for the ensuing mortality rate in WT flies. In other words, along with other physiological indicators for aging progression examined above (Figures 4 and 5B), ID spike counts might also provide a gross forecast of the mortality rate, which is the derivative of the lifespan curve. However, this relationship may not be applicable to a variety of genotypes since it does not seem to hold true for Sod flies (Figure 6A and B).

\section{Signatures of ID Spiking Patterns in Young and Old WT and $\underline{\text { Sod Flies }}$}

Salient features of spike patterns can be uncovered by systematical tracking of the successive changes in consecutive spike intervals to depict the spike train dynamics, as shown in the Poincaré plots (Figure 6F, cf. Lee et al., 2019). In these plots, instantaneous firing frequency, defined as the inverse of an inter-spike interval $\left(\mathrm{ISI}^{-1}\right)$, was first determined for each of the successive spike intervals in a spike train. Each $\mathrm{ISI}^{-1}$ was then plotted against the $\mathrm{ISI}^{-1}$ of the following spike interval and the process was reiterated sequentially for each spike in the ID spike train (i.e. $\mathrm{ISI}^{-1}{ }_{i}$ Vs. $\mathrm{ISI}^{-1}{ }_{i+1}$ for the $i^{\text {th }}$ spike in the sequence). For strictly periodic spiking, all data points of the Poincaré trajectory (PT) would fall at a single location along the diagonal. Irregular deviations from rhythmicity between successive spike intervals appear as perpendicular shifts away from the diagonal. Thus, the trajectory depicts the evolution of spiking frequency, while excursion patterns about the diagonal in the PT indicate characteristics of recurrent firing patterns. Poincaré plots of representative individual spike trains are shown in Figure 6F that readily capture the larger number of spikes with increasing $\mathrm{ISI}^{-1}$ in aged WT flies compared to younger counterparts (up to $100 \mathrm{~Hz}$ vs. $<30 \mathrm{~Hz}$ ). In contrast, the few ID spikes in Sod flies, regardless of age, occurred in a lower frequency range $(\sim 10 \mathrm{~Hz})$.

To integrate information embedded in individual PTs to indicate overall differences in ID spiking dynamics between age groups, we replotted the PTs to carry both frequency and local variation for successive ISIs in order to combine them into an 'average' trajectory (Figure 6G, see Methods and Lee et al., 2019 for computational details). Briefly, for each ISI in the ID spike 
sequence, the ISI ${ }^{-1}$ was plotted against the instantaneous coefficient of variation $\left(\mathrm{CV}_{2}\right)$, defined as: $2\left|\mathrm{ISI}^{-1}{ }_{i}-\mathrm{ISI}^{-1}{ }_{i+1}\right| /\left[\mathrm{ISI}^{-1}{ }_{i}+\mathrm{ISI}^{-1}{ }_{i+1}\right]$ (Holt et al., 1996). Along the trajectory, low $\mathrm{CV}_{2}$ values correspond to more regular firing (lower fractional changes among adjacent spike intervals), while high $\mathrm{CV}_{2}$ values indicate where more abrupt changes in spike intervals occur. The IDs of young WT flies $\left(<1 \%\right.$ mortality) reared at either $25^{\circ} \mathrm{C}$ or $29^{\circ} \mathrm{C}$ displayed rather similar ISI ${ }^{-1}$ vs $\mathrm{CV}_{2}$ trajectories compared to each other, with peak $\mathrm{ISI}^{-1}$ between 10 and $20 \mathrm{~Hz}$ and a similar range of $\mathrm{CV}_{2}$ in the spiking procession. Remarkably, for both rearing temperatures, the trajectories of aged WT flies displayed similar transformation from their young counterparts, with more complex, right-shifted trajectories with peak ISI $^{-1} \mathrm{~s}>70 \mathrm{~Hz}$ (Figure 6G, $41-50 \mathrm{~d}$ and 21-30 d, respectively, for $25^{\circ} \mathrm{C}$ and $29^{\circ} \mathrm{C}$-rearing), suggesting that the changes in circuit activity patterns across lifespan are conserved during high-temperature rearing. Nevertheless, the simple $\mathrm{ISI}^{-1}$ vs $\mathrm{CV}_{2}$ trajectories of both young and aged Sod stood in sharp contrast to WT trajectories, providing another major distinction between the effects of high-temperature and oxidative stressors and underscoring the hypoexcitable nature of the Sod mutant.

Clearly, these "average" trajectories present concisely the overall characteristics of the ID spiking activity, with precise temporal information from the initiation to cessation of the spike train. They are effective signatures for IDs of different age groups in each genotype; WT flies reared at 25 and $29^{\circ} \mathrm{C}$ traverse similar terrains in the span of ID spike trains whereas Sod flies of various ages yield only abbreviated signatures, marking very limited terrains in specific regions in the frequency-variance landscape.

\section{Discussion}

This report summarizes our initial efforts towards a quantitative description of neurophysiological changes during aging in several well-established motor circuits in Drosophila. Aging studies in flies have largely focused on how the lifespan is influenced by genetic modifications in cellular biochemical pathways, such as those regulated by insulin-like peptide (e.g. Clancy et al., 2001; Hwangbo et al., 2004) and target of rapamycin (e.g. Kapahi et al., 2004) signaling and oxidative stress (e.g. Phillips et al., 2000; Tower, 1996), as well as by environmental factors, including diet (e.g. Mair et al., 2003; Piper \& Partridge, 2007; Skorupa et al., 2008) mating experience (Gendron et al., 2014; Harvanek et al., 2017; Kuo et al., 2012), exercise (Piazza et al., 2009; Sujkowski et al., 2015), and trauma (Katzenberger et al., 2015; 
536 Katzenberger et al., 2013). In parallel, several established behavioral assays have provided

537 readouts of age-related functional decline, including: fast phototaxis and negative-geotaxis (e.g.

538 Arking \& Wells, 1990; Miquel et al., 1976; Simon et al., 2006), flight (Miller et al., 2008),

539 memory (Tamura et al., 2003; Yamazaki et al., 2007), courtship (Cook \& Cook, 1975; Partridge et al., 1987), as well as sleep and other circadian activity patterns (Bushey et al., 2010; Koh et al., 2006). However, behavior-relevant studies registering concomitant changes in neurophysiological processes in Drosophila have been largely limited to electroretinogram (Ueda et al., 2018), peripheral neuromuscular synapses (Banerjee et al., 2021), or GF pathway SLR properties (Augustin et al., 2018; Augustin et al., 2019; Blagburn, 2020; Martinez et al., 2007). Our work provides an initial glimpse across an array of motor circuits including the upstream processing of the GF-mediated escape circuit, and pattern generators driving flight and seizure discharges. Furthermore, our observations provide a first-order quantitative comparison of age-trajectories, revealing drastically different effects of high-temperature rearing and oxidative stress on the aging-progression of various neurophysiological parameters in WT and mutant flies.

551

Aging-Resilient and-Vulnerable Aspects of Motor Circuit Function in Drosophila

In this study, we adopted a normalization in time scales of aging progression in accordance with \% mortality (Jones et al., 2014) to facilitate comparisons across the genotypes and conditions that yield drastically different chronological lifespan curves (Figure 1B). A graphical overview emerges when comparing the age-trajectories of the various motor circuit parameters in different fly populations. Relative to the starting point (i.e. youngest age group), changes (in fold on a semi-logarithmic $\log _{2}$ scale) in various circuit functions can be compared directly for their relative vulnerability or resilience to aging (Figure 7).

Our results suggest the operation and integrity of motor circuits fall into two general categories: aging-resilient (Figure 7, lighter shading) and -vulnerable (darker shading). Notably, the elements in the GF pathway that mediate the escape reflex appear more robust and show less functional decline up to the very end of the lifespan and display milder effects of hightemperature stress or ROS scavenger disruption, with little impact on SLR and LLR threshold and latency (Figure 7B-1, -2, -4 and -5). In contrast, substantial changes were seen in the more plastic, use-dependent, functions of these circuit components, including the twin-pulse refractory 
period of the GF pathway and the habituation of its afferent inputs (Figure 7B-3, -6, and -9).

Furthermore, motor program-related DLM spike readouts, such as the flight and ECS discharge patterns, showed remarkable age-related modifications (Fig 7B-8, -10, -11 and -12).

\section{GF Pathway SLRs: GF Neuron and Downstream Elements}

The GF pathway with its well-described neural elements and transmission properties offers an ideal model system to explore cellular mechanisms of aging and neurodegenerative diseases, as previously reported for its SLR properties (Augustin et al., 2018; Martinez et al., 2007; Sofola et al., 2010; Zhao et al., 2010). Consistently, our characterization of SLRs underlying the escape reflex demonstrates robustly maintained basic mechanisms, for signal propagation from the GF neuron through the PSI and DLM motor neuron to postsynaptic DLMs. Over a large portion of the lifespan, including the oldest flies (95\% mortality), WT flies reared at both room 25 and $29^{\circ} \mathrm{C}$-did not show a clear trend of increase or decrease in either SLR latency or threshold, but a decreasing trend in both threshold and latency was noted in Sod mutant flies, indicating a consequence of chronic oxidative stress (Figure 2C, Figure 7B-1 and -2).

More evident aging-vulnerable alterations were seen in the activity-dependent properties for flies grown under high-temperature or ROS stressors. For WT reared at $25{ }^{\circ} \mathrm{C}$, frequencydependent characteristics of the SLR, including the twin-pulse RP and $\mathrm{FF}_{30}$, were relatively stable with no statistically significant age-dependent changes detected (Figure 3A and Table 1). However, this is not the case for $29^{\circ} \mathrm{C}$-reared WT or $S o d$ mutant flies since detectable but opposite trends of changes were observed in these two categories of flies in both twin-pulse RP and $\mathrm{FF}_{30}$ readouts (Figure 3A, Figure 7B-3, Table 1), suggesting differential effects on certain vulnerable properties by these two types of stressors (e.g. mechanisms underlying axonal conduction and/or synaptic transmission).

\section{GF Afferent Processing: LLR Refractory Period and Habituation}

The processing of afferent signals from upstream circuits activates the GF LLRs (Figure 7A). Similar to SLRs, we found activity-dependent properties of LLRs, i.e. RP and habituation were more markedly modified over the lifespan than the subtle changes in latencies and thresholds (Figures 2D, 3B, 4; Table 1; Figure 7B-4 and -5). Given the apparent stability of transmission of the GF neuron and downstream elements, these changes hint at age-dependent 
plasticity in GF afferents in the brain (Engel \& Wu, 1996; 1998), presumably in identified visual (Ache et al., 2019) or mechanosensory (Blagburn, 2020; Lehnert et al., 2013; Mu et al., 2014) inputs.

We observed increasing trends of LLR latency and threshold as flies age (Figures 2D; Table 1; Figure 7B-4 and -5). However, even though age-dependent alterations observed in LLR latency are statistically significant, they are relatively minor in magnitude, constituting only small fractions in time scale within a 5-20 ms behavioral repertoire for visually or olfactorily triggered GF-mediated escape responses (Engel \& Wu, 1992, 1996; Trimarchi \& Schneiderman, 1995a; von Reyn et al., 2017).

We also noted that the LLR RP determined by the paired-pulse protocol revealed substantial heterogeneity in the older WT populations examined as indicated by extremely large spread of data points (50 and $95 \%$ mortality at 25 and $29^{\circ} \mathrm{C}$, Figure 3B, Table 1), indicating the stochastic nature of aging progression in GF afferent signal processing. Importantly, the LLR habituation protocol (Engel \& Wu, 1996; 1998) demonstrated clearest monotonic age-dependent changes in the performance of the GF pathway. In both 25 and $29^{\circ} \mathrm{C}$ reared WT, as well as Sod, populations, aged flies habituated much faster compared to younger counterparts (Figure 4, Figure 7B-9). Consistent across all populations, these effects were evident for all frequencies tested $\left(1,2\right.$, and $5 \mathrm{~Hz}$, with $\mathrm{r}_{\mathrm{s}}$ coefficients ranging from 0.33 to 0.56 and with significant $p$ values). Furthermore, habituation is commonly considered a simple form of non-associative learning (Engel \& Wu, 2009; Rankin et al., 2009). Our observation that the Drosophila GF habituation rate increases monotonically and robustly with age (Figures 4 and 7B-9) renders this plasticity parameter a potential quantitative index of aging progression for an important aspect of nervous system function.

\section{$\underline{\text { Flight }}$}

Throughout most of their lifespan, WT flies maintained their flight ability (Figure 1C) with stable WBFs (Figure 1D, F; Figure 7B-7), suggesting the presence of effective homeostatic adjustments to overcome aging-associated changes in biomechanical properties of flight muscles and thoracic case. As previously reported (Miller et al., 2008), WBF is stable beyond the median lifespan in spite of marked changes in muscle structure and stiffness (see also Chaturvedi et al., 2019). Despite the nearly invariant WBFs, we found the flight pattern generator circuit driving 
629 DLM displayed a clear and largely monotonic increase in firing rate with age (Figure 1E, Figure 630 7B-8), a robust phenotypic change in both $25^{\circ} \mathrm{C}$ and $29^{\circ} \mathrm{C}$-reared flies. Conceivably, the age631 dependent increases in firing rate could indicate changes in motoneuron (DLMn) excitability or 632 flight patterning circuit signal processing among the relevant thoracic interneurons. In either 633 case, this provides a compensatory mechanism to ensure flight ability as aging progresses, 634 namely, increasing the firing rate of muscle $\mathrm{Ca}^{2+}$ action potential to retain appropriate 635 intracellular $\mathrm{Ca}^{2+}$ levels for powering sustained flight in myogenic stretch-activated DLMs 636 (Gordon \& Dickinson, 2006; Lehmann et al., 2013; Pringle, 1978).

\section{Electroconvulsive Seizure}

In tethered flies, high frequency stimulation across the brain recruits a remarkably stereotypic pattern of nervous system-wide spike discharges accompanied by wing-buzzes (in a fixed succession of ID, paralysis, and DD, cf. Lee \& Wu, 2002), reminiscent of the sequence of seizures and paralysis induced by mechanical shock in "bang-sensitive" mutants (Burg \& Wu, 2012; Ganetzky \& Wu, 1982). Importantly, the spiking associated with the ID and DD is independent of the GF system; during the ECS repertoire, transmission along the escape pathway is blocked (Pavlidis \& Tanouye, 1995). Analysis of decapitated flies and Ring-X gynandromorph mosaics has further indicated that both ID and DD pattern generators reside in the thorax, although globally synchronized electric activities and quiescence episodes concurrent with the ID-paralysis-DD repertoire also emerge along the body axis (Lee \& Wu, 2002) and across the brain (Iyengar \& Wu, 2021). However, the ID and DD are likely derived from separable network origins, as they are independently vulnerable to different ion channel mutations (Kasuya et al., 2019; Lee \& Wu, 2006).

In both WT and Sod flies, we noted a clear age-dependent decrease in the threshold of ECS-evoked seizure across the lifespan (Figure 5, Figure 7B-10). The monotonic characteristic of this reduction suggests seizure threshold may be considered as another quantitative index of the age progression in nervous system function. Indeed, aged rats reportedly show reduced threshold for kainate-induced seizures (Liang et al., 2007) and elderly humans display increased incidence of seizure disorders (Leppik et al., 2006; Tallis et al., 1991). and -12), most dramatically during the ID phase (Figure 6). Instead of a monotonic trend, ID 
spiking increases initially with age, peaking around 50\% mortality, then declines to earlier levels towards the end of the lifespan (Figures 6B and 7B-11). It is also intriguing to see that changing ID spiking intensity seemingly provides a forecast for subsequent mortality rate. Indeed, with appropriate lags ( $11 \mathrm{~d}$. and $6 \mathrm{~d}$. For 25 and $29^{\circ} \mathrm{C}$-reared flies), there are high degrees of crosscorrelation between the two parameters (Figure $6 \mathrm{~F} \& \mathrm{G}$ ). It would be interesting to examine this relationship in additional mutants with intrinsically altered neuronal excitability and lifespan. For example, both hyper-excitable Shaker $(S h)$ and hypo-excitable mle $e^{\text {napts }}\left(\right.$ nap $^{\text {ts }}$ ) mutants display shortened lifespans (Reenan \& Rogina, 2008; Trout \& Kaplan, 1970) along with drastically altered ECS discharges (Lee \& Wu, 2006), flight pattern generation (Iyengar \& Wu, 2014, unpublished observations), and habituation (Engel \& Wu, 1998).

\section{Distinctive Patterns of Aging-Related Changes by High Temperature and Oxidative Stress}

The distinct aging trajectories manifested in the functioning of the various motor circuits described above clearly delineate the manners in which high-temperature rearing and oxidative stress, imposed by external environment versus internal milieu, differ in their effects on aging progression in motor functions.

Ambient temperature is a key factor in determining lifespan in Drosophila (Loeb \& Northrop, 1917) and other ectotherms. Rearing at high temperature $\left(29^{\circ} \mathrm{C}\right)$, lifespan is shortened by $\sim 40-50 \%$ compared to $25{ }^{\circ} \mathrm{C}$, (Figure 1B). Our data support the notion that aging progression is faster at $29^{\circ} \mathrm{C}$ but it largely retains the characteristics of the motor performance trajectories of $25^{\circ} \mathrm{C}$, as though it proceeds at a "compressed" time scale. Parameters that were age-resilient in $25{ }^{\circ} \mathrm{C}$-reared flies were robustly maintained in $29^{\circ} \mathrm{C}$-reared individuals (SLR and LLR latency and threshold, flight WBF, Figure 7B lighter shade). Similarly, age-vulnerable parameters generally preserved consistent trends in age-dependent changes when scaled for $\%$ mortality. This is true particularly for increased DLM firing frequency during flight, increased LLR habituation rate, and decreased ECS seizure threshold (Figure 7B-8, -9, -10 darker shade). Remarkably, even the bell-shaped age profile of ID spiking was retained in the $29^{\circ} \mathrm{C}$-reared WT population (Figure 7B-11). For the remaining parameters, SLR and LLR refractory periods, habituation and DD spiking, $29^{\circ} \mathrm{C}$-rearing appeared to intensify their age-dependence, leading to considerably steeper trends (Figure 7B-3, -6, -12). Our findings suggest that in the shortened lifespans of temperature-stressed individuals, the temporal characteristics of neurophysiological 
691

692

693

694

695

696

697

698

699

700

701

702

703

704

705

706

707

708

709

710

711

712

713

714

715

716

717

718

719

720

721

changes is largely retained, albeit "accelerated" according to the degree of lifespan compression.

Importantly, these observations give justification for the common practice of using $29{ }^{\circ} \mathrm{C}$-rearing to expedite experiments in Drosophila aging studies.

Our findings from Sod mutants indicate that oxidative stress exerts strong influences on some physiological parameters with outcomes distinct from the effects of high-temperature rearing. Oxidative stress, resulting from inefficient clearance of metabolic reactive oxygen species (ROS, e.g. superoxide anion and other free radical species), is thought as a major contributor to aging processes (Finkel \& Holbrook, 2000; Harman, 1956; Harman, 1981). A key class of ROS scavenging enzymes is the Superoxide dismutases which convert superoxide anions into hydrogen peroxide (Hart et al., 1999; Miller, 2012). In Drosophila, three Superoxide dismutase (Sod) enzymes have been identified: intracellular $\mathrm{Cu}^{2+} / \mathrm{Zn}^{2+}$ Sod1 (encoded by Sod, Phillips et al., 1989), mitochondrial $\mathrm{Mn}^{2+}$ Sod2 (encoded by Sod2, Duttaroy et al., 2003; also known as bewildered, Celotto et al., 2012), or extracellular Sod3 (encoded by Sod3, Jung et al., 2011 ). Stress resistance and longevity of the fly are greatly compromised when any of the three is disrupted. Indeed, the $\operatorname{Sod}$ allele described in this study has no detectable enzymatic activity (Phillips et al., 1989), and exhibits locomotor defects as well as sensitivity to mechanical-, paraquat- and heat-stress along with greatly shortened lifespan (Ruan \& Wu, 2008).

Across the Sod populations, the general trend still holds true in that the identified ageresilient parameters could sustain functionality throughout the lifespan (GF SLR and LLR latency and threshold, flight WBF, Figure 7B lighter shade). For several aging-vulnerable properties, Sod flies also follow the general profile of age progression albeit with modified amplitude of expression. These are apparent in DLM firing during flight and ID spiking (Figure 7B-8, -11), and more notably, in both accelerated rate of habituation and reduced ECS seizure threshold, closely resembling the corresponding age-trajectories in WT counterparts (Figure 7B9 and -10). Nevertheless, more striking alterations in Sod are seen in the activity-dependent properties of the GF pathway, as reflected in the trajectories of refractory period for both SLR and LLR that are clearly distinct from high-temperature effects (Figure 7B-3, -6).

A unique feature of the aging trajectories of Sod motor functions is the apparent anomaly in some age groups that exhibit unexpected trends in phenotypic extremity. Among different age groups of Sod mutants, the youngest age group ( $<5 \%$ mortality) displayed the most conspicuous deviations from the WT counterpart in several categories of motor performance (Figure 7B), 
722

including diminished flight ability (Figure 1C), longer SLR latency (Figure 2C), lengthened SLR and LLR refractory periods (RP, Figure 3), increased ECS threshold (Figure 5B), and decreased spiking during seizure discharges (Figures $5 \mathrm{C} \& 6 \mathrm{~B}$ ). These parameters were often accompanied by a greater spread in measurements among the youngest $\operatorname{Sod}$ flies (e.g. Figures $3 \mathrm{~A} \& \mathrm{~B} ; 5 \mathrm{~B}$ ). Intriguingly, the aged $\operatorname{Sod}$ flies ( $70 \& 95 \%$ mortality) paradoxically performed at levels comparable to their aged WT counterparts (Figure 7B), as though they could outperform the young mutant flies. These findings are shared by an independently isolated loss-of-function Sod allele ( $S \theta d^{21}$, Ueda, Iyengar \& Wu, 2021). It is possible that secondary mutations in the red genetic background contribute to the observed differences between the Sod mutants and their WT counterparts, as genetic background effects can have profound effects on specific aging phenotypes (Partridge \& Gems, 2007). However, observations from an independently generated loss-of-function $\operatorname{Sod}$ allele $\left(\operatorname{Sod}^{21}\right)$ revealed a similar set of GF transmission phenotypes in young $\operatorname{Sod}^{21}$ flies (<5\% mortality), including a retarded SLR response latency and a poor ability for SLRs to follow high-frequency stimulation (Ueda et al., 2021). Thus, unidentified modifiers in the background are an unlikely explanation for the respective Sod phenotypes reported here, which can be seen even in the youngest adult Sod populations.

A likely explanation for this seemingly counterintuitive trend could be the highly stochastic nature of oxidative insults in both development and function of neural circuits such that a portion Sod flies carry more severe but variable in-born defects, compounding the manifestation of aging progression. distinct from aging processes. Therefore, the Sod mutant population undergoes a progressive selection over time in favor of healthier individuals while those with poor circuit performance die off earlier. Consistent with this view, Sod mutants reportedly have difficulty eclosing from their pupal case (Phillips et al., 1989; Şahin et al., 2017; Woods, 2017) and the Sod lifespan curve lacks the earlier plateau phase (Figure 1B) resulting from much higher mortality in young individuals compared to WT populations. This possibility is further highlighted in a recent study demonstrating certain differences in functional consequences of oxidative stress imposed chronically throughout development in mutant flies and those resulting from acute induction by drug feeding to healthy flies. In WT flies, elevated ROS levels induced by paraquat feeding lead to a distinct collection GF transmission phenotypes compared to Sod mutants (Ueda et al., 2021). For example, the SLR latency and refractory period defects in Sod mutants are not observed in paraquat-fed WT flies. 
Physiological Hallmarks of Aging Progression in Motor Circuits: Quantitative Biomarkers

It is desirable to search for potential physiological aging indices to completement existing cellular and molecular biomarkers of aging in Drosophila, such as erroneous expression of the transcription factor genes wingless and engrailed (Rogina et al., 1997), accumulation of glycation end-products (Baynes, 2001; Jacobson et al., 2010; Oudes et al., 1998) and heat-shock protein (Landis et al., 2004; Tower, 2011), as well as modified functional states of the insulin and TOR signaling pathways components (Kapahi et al., 2004; Partridge et al., 2011).

Among the various age-dependent trajectories in motor circuit performance, two protocols, LLR habituation and ECS induction, yielded parameters that displayed remarkably consistent aging progressions in normal, high temperature-reared and ROS-stressed populations. Specifically, both acceleration of the habituation rate (Figures 4) and reduction in ECS seizure threshold (Figures 5B) displayed a relatively uniform, monotonic age-trajectories (Figure 7B-9 and -10), providing an unambiguous read-out of the circuit performance index along the percent mortality axis. It will be particularly interesting to examine whether the age-trajectories of these parameters are similarly scaled against lifespan in long-lived flies such as WT flies under calorie restriction conditions or in chico and other insulin pathway mutants. Furthermore, future studies can utilize these physiological properties to study the relationships between life-span and healthspan in the context of various environmental conditions, pharmacological interventions or genetic manipulations which affect longevity.

Future studies will further evaluate their suitability as readily accessible, quantitative indices for more incisive aging assessment in WT and mutant flies. This will be a desirable addition in aging research using a wealth of available mutants, such as insulin signaling pathway mutants that are known to affect lifespan, under various environmental conditions, 


\section{References}

Allen, M. J., \& Murphey, R. (2007). The chemical component of the mixed GF-TTMn synapse in Drosophila melanogaster uses acetylcholine as its neurotransmitter. European Journal of Neuroscience, 26(2), 439-445.

Arking, R., \& Wells, R. A. (1990). Genetic alteration of normal aging processes is responsible for extended longevity in Drosophila. Developmental Genetics, 11(2), 141-148. doi:10.1002/dvg.1020110204

Atwood, H. (1992). Age-dependent alterations of synaptic performance and plasticity in crustacean motor systems. Experimental Gerontology, 27(1), 51-61.

Augustin, H., McGourty, K., Allen, M. J., Adcott, J., Wong, C. T., Boucrot, E., \& Partridge, L. (2018). Impact of insulin signaling and proteasomal activity on physiological output of a neuronal circuit in aging Drosophila melanogaster. Neurobiology of Aging, 66, 149-157. doi:10.1016/j.neurobiolaging.2018.02.027

Augustin, H., Zylbertal, A., \& Partridge, L. (2019). A Computational Model of the Escape Response Latency in the Giant Fiber System of Drosophila melanogaster. Eneuro, 6(2). doi:10.1523/eneuro.0423-18.2019

Banerjee, S., Vernon, S., Jiao, W., Choi, B. J., Ruchti, E., Asadzadeh, J., . . McCabe, B. D. (2021). Miniature neurotransmission is required to maintain Drosophila synaptic structures during ageing. Nature Communications, 12(1), 4399. doi:10.1038/s41467-02124490-1

Baynes, J. W. (2001). The role of AGEs in aging: causation or correlation. Experimental Gerontology, 36(9), 1527-1537. doi:10.1016/s0531-5565(01)00138-3

Biteau, B., Karpac, J., Supoyo, S., DeGennaro, M., Lehmann, R., \& Jasper, H. (2010). Lifespan extension by preserving proliferative homeostasis in Drosophila. Plos Genetics, 6(10), e1001159.

Blagburn, J. M. (2020). A new method of recording from the giant fiber of Drosophila melanogaster shows that the strength of its auditory inputs remains constant with age. PloS One, 15(1), e0224057. doi:10.1371/journal.pone.0224057

Blagburn, J. M., Alexopoulos, H., Davies, J. A., \& Bacon, J. P. (1999). Null mutation in shakingB eliminates electrical, but not chemical, synapses in the Drosophila giant fiber system: a structural study. Journal of Comparative Neurology, 404(4), 449-458.

Burg, M. G., \& Wu, C. F. (2012). Mechanical and temperature stressor-induced seizure-andparalysis behaviors in Drosophila bang-sensitive mutants. Journal of Neurogenetics, 26(2), 189-197. doi:10.3109/01677063.2012.690011

Bushey, D., Hughes, K. A., Tononi, G., \& Cirelli, C. (2010). Sleep, aging, and lifespan in Drosophila. BMC Neuroscience, 11, 56. doi:10.1186/1471-2202-11-56

Campbell, S. D., Hilliker, A. J., \& Phillips, J. P. (1986). Cytogenetic analysis of the cSOD microregion in Drosophila melanogaster. Genetics, 112(2), 205-215.

Celotto, A. M., Liu, Z., Vandemark, A. P., \& Palladino, M. J. (2012). A novel Drosophila SOD2 mutant demonstrates a role for mitochondrial ROS in neurodevelopment and disease. Brain Behav, 2(4), 424-434. doi:10.1002/brb3.73

Chan, W. P., \& Dickinson, M. H. (1996). In vivo length oscillations of indirect flight muscles in the fruit fly Drosophila virilis. Journal of Experimental Biology, 199(Pt 12), 2767-2774. 
Chaturvedi, D., Prabhakar, S., Aggarwal, A., Atreya, K. B., \& VijayRaghavan, K. (2019). Adult Drosophila muscle morphometry through microCT reveals dynamics during ageing. Open Biol, 9(6), 190087. doi:10.1098/rsob.190087

Clancy, D. J., Gems, D., Harshman, L. G., Oldham, S., Stocker, H., Hafen, E., . . Partridge, L. (2001). Extension of life-span by loss of CHICO, a Drosophila insulin receptor substrate protein. Science, 292(5514), 104-106. doi:10.1126/science.1057991

Cook, R., \& Cook, A. (1975). The attractiveness to males of female Drosophila melanogaster: effects of mating, age and diet. Animal Behaviour, 23(3), 521-526. doi:10.1016/00033472(75)90129-3

Curtsinger, J. W., Fukui, H. H., Khazaeli, A. A., Kirscher, A., Pletcher, S. D., Promislow, D. E., \& Tatar, M. (1995). Genetic variation and aging. Annual Review of Genetics, 29(1), 553575.

Curtsinger, J. W., Fukui, H. H., Townsend, D. R., \& Vaupel, J. W. (1992). Demography of genotypes: failure of the limited life-span paradigm in Drosophila melanogaster. Science, 258(5081), 461-463. doi:10.1126/science.1411541

Dickinson, M. H. (2014). Death Valley, Drosophila, and the Devonian toolkit. Annual Review of Entomology, 59, 51-72. doi:10.1146/annurev-ento-011613-162041

Dickinson, M. H., \& Tu, M. S. (1997). The function of dipteran flight muscle. Comparative Biochemistry and Physiology Part A: Physiology, 116(3), 223-238.

Duttaroy, A., Paul, A., Kundu, M., \& Belton, A. (2003). A Sod2 null mutation confers severely reduced adult life span in Drosophila. Genetics, 165(4), 2295-2299.

Efron, B. (1981). Nonparametric estimates of standard error: the jackknife, the bootstrap and other methods. Biometrika, 68(3), 589-599.

Elkins, T., \& Ganetzky, B. (1990). Conduction in the giant nerve fiber pathway in temperaturesensitive paralytic mutants of Drosophila. Journal of Neurogenetics, 6(4), 207-219. doi:10.3109/01677069009107111

Engel, J. E., \& Wu, C.-F. (1992). Interactions of membrane excitability mutations affecting potassium and sodium currents in the flight and giant fiber escape systems of Drosophila. Journal of Comparative Physiology A, 171(1), 93-104.

Engel, J. E., \& Wu, C.-F. (1996). Altered habituation of an identified escape circuit in Drosophila memory mutants. Journal of Neuroscience, 16(10), 3486-3499.

Engel, J. E., \& Wu, C. F. (1998). Genetic dissection of functional contributions of specific potassium channel subunits in habituation of an escape circuit in Drosophila. Journal of Neuroscience, 18(6), 2254-2267. doi:10.1523/jneurosci.18-06-02254.1998

Engel, J. E., \& Wu, C. F. (2009). Neurogenetic approaches to habituation and dishabituation in Drosophila. Neurobiology of Learning and Memory, 92(2), 166-175. doi:10.1016/j.nlm.2008.08.003

Engel, J. E., Xie, X.-J., Sokolowski, M. B., \& Wu, C.-F. (2000). A cGMP-dependent protein kinase gene, foraging, modifies habituation-like response decrement of the giant fiber escape circuit in Drosophila. Learning \& Memory, 7(5), 341-352.

Finkel, T., \& Holbrook, N. J. (2000). Oxidants, oxidative stress and the biology of ageing. Nature, 408(6809), 239-247.

Frankel, A., \& Brousseau, G. (1968). Drosophila medium that does not require dried yeast. Drosophila Information Service, 43, 184. 
Ganetzky, B., \& Wu, C. F. (1982). Indirect Suppression Involving Behavioral Mutants with Altered Nerve Excitability in DROSOPHILA MELANOGASTER. Genetics, 100(4), 597-614.

Gargano, J. W., Martin, I., Bhandari, P., \& Grotewiel, M. S. (2005). Rapid iterative negative geotaxis (RING): a new method for assessing age-related locomotor decline in Drosophila. Experimental Gerontology, 40(5), 386-395.

Gendron, C. M., Kuo, T. H., Harvanek, Z. M., Chung, B. Y., Yew, J. Y., Dierick, H. A., \& Pletcher, S. D. (2014). Drosophila life span and physiology are modulated by sexual perception and reward. Science, 343(6170), 544-548. doi:10.1126/science.1243339

Gorczyca, M., \& Hall, J. C. (1984). Identification of a cholinergic synapse in the giant fiber pathway of Drosophila using conditional mutations of acetylcholine synthesis. Journal of Neurogenetics, 1(4), 289-313.

Gordon, S., \& Dickinson, M. H. (2006). Role of calcium in the regulation of mechanical power in insect flight. Proceedings of the National Academy of Sciences of the United States of America, 103(11), 4311-4315. doi:10.1073/pnas.0510109103

Götz, K. G. (1968). Flight control in Drosophila by visual perception of motion. Kybernetik, 4(6), 199-208. doi:10.1007/bf00272517

Götz, K. G. (1987). Course-control, metabolism and wing interference during ultralong tethered flight in Drosophila melanogaster. Journal of Experimental Biology, 128(1), 35-46.

Harcombe, E. S., \& Wyman, R. J. (1977). Output pattern generation by Drosophila flight motoneurons. Journal of Neurophysiology, 40(5), 1066-1077.

Harman, D. (1956). Aging: A Theory Based on Free Radical and Radiation Chemistry. Journal of Gerontology, 11(3), 298-300. doi:10.1093/geronj/11.3.298

Harman, D. (1981). The aging process. Proceedings of the National Academy of Sciences of the United States of America, 78(11), 7124-7128. doi:10.1073/pnas.78.11.7124

Hart, P. J., Balbirnie, M. M., Ogihara, N. L., Nersissian, A. M., Weiss, M. S., Valentine, J. S., \& Eisenberg, D. (1999). A structure-based mechanism for copper- zinc superoxide dismutase. Biochemistry, 38(7), 2167-2178.

Harvanek, Z. M., Lyu, Y., Gendron, C. M., Johnson, J. C., Kondo, S., Promislow, D. E. L., \& Pletcher, S. D. (2017). Perceptive costs of reproduction drive ageing and physiology in male Drosophila. Nat Ecol Evol, 1(6), 152. doi:10.1038/s41559-017-0152

Heisenberg, M., \& Wolf, R. (1984). Visual Control in Free Flight. In Vision in Drosophila (pp. 180-182). Berlin: Heidelberg.

Holt, G. R., Softky, W. R., Koch, C., \& Douglas, R. J. (1996). Comparison of discharge variability in vitro and in vivo in cat visual cortex neurons. Journal of Neurophysiology, 75(5), 1806-1814. doi:10.1152/jn.1996.75.5.1806

Hwangbo, D. S., Gershman, B., Tu, M. P., Palmer, M., \& Tatar, M. (2004). Drosophila dFOXO controls lifespan and regulates insulin signalling in brain and fat body. Nature, 429(6991), 562-566. doi:10.1038/nature02549

Iyengar, A., Ruan, H., \& Wu, C.-F. (2010). Physiological correlates of social interactionmediated lifespan extension in sensory-motor integration of Drosophila Sod mutants. Paper presented at the Society for Neuroscience Annual Meeting, Washington DC.

Iyengar, A., \& Wu, C.-F. (2014). Flight and seizure motor patterns in Drosophila mutants: simultaneous acoustic and electrophysiological recordings of wing beats and flight muscle activity. Journal of Neurogenetics, 28(3-4), 316-328. 
Iyengar, A., \& Wu, C.-F. (2021). Fly seizure EEG: field potential activity in the Drosophila brain. Journal of Neurogenetics, 35(3), 295-305. doi:10.1080/01677063.2021.1950714

Iyengar, A. S. R. (2016). An integrative analysis of neuronal hyperexcitability, central pattern generation and aberrant motor behavior through the lens of Drosophila neurogenetics. (PhD). University of Iowa, Iowa City.

Jacobson, J., Lambert, A. J., Portero-Otín, M., Pamplona, R., Magwere, T., Miwa, S., . . Partridge, L. (2010). Biomarkers of aging in Drosophila. Aging Cell, 9(4), 466-477. doi:10.1111/j.1474-9726.2010.00573.x

Janse, C., Van der Roest, M., \& Slob, W. (1986). Age-related decrease in electrical coupling of two identified neurons in the mollusc Lymnaea stagnalis. Brain Research, 376(1), 208212.

Jones, O. R., Scheuerlein, A., Salguero-Gómez, R., Camarda, C. G., Schaible, R., Casper, B. B., . . . Vaupel, J. W. (2014). Diversity of ageing across the tree of life. Nature, 505(7482), 169-173. doi:10.1038/nature 12789

Jung, I., Kim, T. Y., \& Kim-Ha, J. (2011). Identification of Drosophila SOD3 and its protective role against phototoxic damage to cells. FEBS Letters, 585(12), 1973-1978. doi:10.1016/j.febslet.2011.05.033

Kang, H.-L., Benzer, S., \& Min, K.-T. (2002). Life extension in Drosophila by feeding a drug. Proceedings of the National Academy of Sciences, 99(2), 838-843.

Kapahi, P., Zid, B. M., Harper, T., Koslover, D., Sapin, V., \& Benzer, S. (2004). Regulation of lifespan in Drosophila by modulation of genes in the TOR signaling pathway. Current Biology, 14(10), 885-890. doi:10.1016/j.cub.2004.03.059

Kasuya, J., Iyengar, A., Chen, H.-L., Lansdon, P., Wu, C.-F., \& Kitamoto, T. (2019). Milk-whey diet substantially suppresses seizure-like phenotypes of paraShu, a Drosophila voltagegated sodium channel mutant. Journal of Neurogenetics, 33(3), 164-178.

Katzenberger, R. J., Chtarbanova, S., Rimkus, S. A., Fischer, J. A., Kaur, G., Seppala, J. M., . . . Wassarman, D. A. (2015). Death following traumatic brain injury in Drosophila is associated with intestinal barrier dysfunction. Elife, 4. doi:10.7554/eLife.04790

Katzenberger, R. J., Loewen, C. A., Wassarman, D. R., Petersen, A. J., Ganetzky, B., \& Wassarman, D. A. (2013). A Drosophila model of closed head traumatic brain injury. Proceedings of the National Academy of Sciences of the United States of America, 110(44), E4152-4159. doi:10.1073/pnas.1316895110

Kenyon, C. (2001). A conserved regulatory system for aging. Cell, 105(2), 165-168.

King, D. G., \& Wyman, R. J. (1980). Anatomy of the giant fibre pathway in Drosophila. I. Three thoracic components of the pathway. Journal of Neurocytology, 9(6), 753-770. doi:10.1007/bf01205017

Koh, K., Evans, J. M., Hendricks, J. C., \& Sehgal, A. (2006). A Drosophila model for ageassociated changes in sleep:wake cycles. Proceedings of the National Academy of Sciences of the United States of America, 103(37), 13843-13847. doi:10.1073/pnas.0605903103

Kuebler, D., \& Tanouye, M. A. (2000). Modifications of seizure susceptibility in Drosophila. Journal of Neurophysiology, 83(2), 998-1009.

Kuo, T. H., Yew, J. Y., Fedina, T. Y., Dreisewerd, K., Dierick, H. A., \& Pletcher, S. D. (2012). Aging modulates cuticular hydrocarbons and sexual attractiveness in Drosophila melanogaster. Journal of Experimental Biology, 215(Pt 5), 814-821. doi:10.1242/jeb.064980 
Landis, G. N., Abdueva, D., Skvortsov, D., Yang, J., Rabin, B. E., Carrick, J., . . Tower, J. (2004). Similar gene expression patterns characterize aging and oxidative stress in Drosophila melanogaster. Proceedings of the National Academy of Sciences of the United States of America, 101(20), 7663-7668. doi:10.1073/pnas.0307605101

Lee, J., Iyengar, A., \& Wu, C.-F. (2019). Distinctions among electroconvulsion-and proconvulsant-induced seizure discharges and native motor patterns during flight and grooming: Quantitative spike pattern analysis in Drosophila flight muscles. Journal of Neurogenetics, 33(2), 125-142.

Lee, J., \& Wu, C.-F. (2002). Electroconvulsive seizure behavior in Drosophila: analysis of the physiological repertoire underlying a stereotyped action pattern in bang-sensitive mutants. Journal of Neuroscience, 22(24), 11065-11079.

Lee, J., \& Wu, C.-F. (2006). Genetic modifications of seizure susceptibility and expression by altered excitability in Drosophila $\mathrm{Na}+$ and $\mathrm{K}+$ channel mutants. Journal of Neurophysiology, 96(5), 2465-2478.

Leffelaar, D., \& Grigliatti, T. (1983). Age-dependent behavior loss in adult Drosophila melanogaster. Developmental Genetics, 4(3), 211-227.

Lehmann, F. O., \& Dickinson, M. H. (1997). The changes in power requirements and muscle efficiency during elevated force production in the fruit fly Drosophila melanogaster. Journal of Experimental Biology, 200(Pt 7), 1133-1143.

Lehmann, F. O., Skandalis, D. A., \& Berthé, R. (2013). Calcium signalling indicates bilateral power balancing in the Drosophila flight muscle during manoeuvring flight. Journal of The Royal Society Interface, 10(82), 20121050.

Lehnert, B. P., Baker, A. E., Gaudry, Q., Chiang, A. S., \& Wilson, R. I. (2013). Distinct roles of TRP channels in auditory transduction and amplification in Drosophila. Neuron, 77(1), 115-128. doi:10.1016/j.neuron.2012.11.030

Leppik, I. E., Kelly, K. M., deToledo-Morrell, L., Patrylo, P. R., DeLorenzo, R. J., Mathern, G. W., \& White, H. S. (2006). Basic research in epilepsy and aging. Epilepsy Research, 68 Suppl 1, S21-37. doi:10.1016/j.eplepsyres.2005.07.014

Levine, J. (1973). Properties of the nervous system controlling flight inDrosophila melanogaster. Journal of comparative physiology, 84(2), 129-166.

Liang, L., Beaudoin, M., Fritz, M., Fulton, R., \& Patel, M. (2007). Kainate-induced seizures, oxidative stress and neuronal loss in aging rats. Neuroscience, 147(4), 1114-1118.

Loeb, J., \& Northrop, J. H. (1917). What Determines the Duration of Life in Metazoa? Proceedings of the National Academy of Sciences of the United States of America, 3(5), 382-386. doi:10.1073/pnas.3.5.382

Mair, W., Goymer, P., Pletcher, S. D., \& Partridge, L. (2003). Demography of dietary restriction and death in Drosophila. Science, 301(5640), 1731-1733.

Martinez, V., Javadi, C., Ngo, E., Ngo, L., Lagow, R., \& Zhang, B. (2007). Age-related changes in climbing behavior and neural circuit physiology in Drosophila. Developmental Neurobiology, 67(6), 778-791.

Miller, A.-F. (2012). Superoxide dismutases: ancient enzymes and new insights. FEBS Letters, $586(5), 585-595$.

Miller, A. (1950). The internal anatomy and histology of the imago of Drosophila melanogaster. In M. Demeric (Ed.), The Biology of Drosophila (pp. 421-534). New York: John Wiley and Sons. 
1004

1005

1006

1007

1008

1009

1010

1011

1012

1013

1014

1015

1016

1017

1018

1019

1020

1021

1022

1023

1024

1025

1026

1027

1028

1029

1030

1031

1032

1033

1034

1035

1036

1037

1038

1039

1040

1041

1042

1043

1044

1045

1046

1047

1048

Miller, M. S., Lekkas, P., Braddock, J. M., Farman, G. P., Ballif, B. A., Irving, T. C., . . . Vigoreaux, J. O. (2008). Aging enhances indirect flight muscle fiber performance yet decreases flight ability in Drosophila. Biophysical Journal, 95(5), 2391-2401. doi:10.1529/biophysj.108.130005

Min, \& Benzer, S. (1997). Spongecake and eggroll: two hereditary diseases in Drosophila resemble patterns of human brain degeneration. Current Biology, 7(11), 885-888.

Miquel, J., Lundgren, P. R., Bensch, K. G., \& Atlan, H. (1976). Effects of temperature on the life span, vitality and fine structure of Drosophila melanogaster. Mechanisms of Ageing and Development, 5, 347-370.

Mu, L., Bacon, J. P., Ito, K., \& Strausfeld, N. J. (2014). Responses of Drosophila giant descending neurons to visual and mechanical stimuli. Journal of Experimental Biology, 217(Pt 12), 2121-2129. doi:10.1242/jeb.099135

Oudes, A. J., Herr, C. M., Olsen, Y., \& Fleming, J. E. (1998). Age-dependent accumulation of advanced glycation end-products in adult Drosophila melanogaster. Mechanisms of Ageing and Development, 100(3), 221-229. doi:10.1016/s0047-6374(97)00146-2

Partridge, L., Alic, N., Bjedov, I., \& Piper, M. D. (2011). Ageing in Drosophila: the role of the insulin/Igf and TOR signalling network. Experimental Gerontology, 46(5), 376-381. doi:10.1016/j.exger.2010.09.003

Partridge, L., \& Gems, D. (2007). Benchmarks for ageing studies. Nature, 450(7167), 165-167. doi:10.1038/450165a

Partridge, L., Hoffmann, A., \& Jones, J. (1987). Male size and mating success in Drosophila melanogaster and D. pseudoobscura under field conditions. Animal Behaviour, 35(2), 468-476.

Pavlidis, P., \& Tanouye, M. A. (1995). Seizures and failures in the giant fiber pathway of Drosophila bang-sensitive paralytic mutants. Journal of Neuroscience, 15(8), 5810-5819.

Phillips, J. P., Campbell, S. D., Michaud, D., Charbonneau, M., \& Hilliker, A. J. (1989). Null mutation of copper/zinc superoxide dismutase in Drosophila confers hypersensitivity to paraquat and reduced longevity. Proceedings of the National Academy of Sciences, 86(8), 2761-2765.

Phillips, J. P., Parkes, T. L., \& Hilliker, A. J. (2000). Targeted neuronal gene expression and longevity in Drosophila. Experimental Gerontology, 35(9-10), 1157-1164. doi:10.1016/s0531-5565(00)00117-0

Piazza, N., Gosangi, B., Devilla, S., Arking, R., \& Wessells, R. (2009). Exercise-training in young Drosophila melanogaster reduces age-related decline in mobility and cardiac performance. PloS One, 4(6), e5886. doi:10.1371/journal.pone.0005886

Piper, M. D., \& Partridge, L. (2007). Dietary restriction in Drosophila: delayed aging or experimental artefact? Plos Genetics, 3(4), e57. doi:10.1371/journal.pgen.0030057

Pletcher, S. D., Macdonald, S. J., Marguerie, R., Certa, U., Stearns, S. C., Goldstein, D. B., \& Partridge, L. (2002). Genome-wide transcript profiles in aging and calorically restricted Drosophila melanogaster. Current Biology, 12(9), 712-723.

Pringle, J. W. S. (1978). The Croonian Lecture, 1977-Stretch activation of muscle: function and mechanism. Proceedings of the Royal Society of London. Series B. Biological Sciences, 201(1143), 107-130.

Rankin, C. H., Abrams, T., Barry, R. J., Bhatnagar, S., Clayton, D. F., Colombo, J., . . . Thompson, R. F. (2009). Habituation revisited: an updated and revised description of the 
1049

1050

1051

1052

1053

1054

1055

1056

1057

1058

1059

1060

1061

1062

1063

1064

1065

1066

1067

1068

1069

1070

1071

1072

1073

1074

1075

1076

1077

1078

1079

1080

1081

1082

1083

1084

1085

1086

1087

1088

1089

1090

1091

1092

1093 behavioral characteristics of habituation. Neurobiology of Learning and Memory, 92(2), 135-138. doi:10.1016/j.nlm.2008.09.012

Reenan, R. A., \& Rogina, B. (2008). Acquired temperature-sensitive paralysis as a biomarker of declining neuronal function in aging Drosophila. Aging Cell, 7(2), 179-186. doi:10.1111/j.1474-9726.2008.00368.X

Rogina, B., Benzer, S., \& Helfand, S. L. (1997). Drosophila drop-dead mutations accelerate the time course of age-related markers. Proceedings of the National Academy of Sciences of the United States of America, 94(12), 6303-6306. doi:10.1073/pnas.94.12.6303

Ruan, H. (2008). On Drosophila aging: Lifespan plasticity, social-behavioral influences, and neurophysiological indices: The University of Iowa.

Ruan, H., \& Wu, C.-F. (2009). Neurophysiological indices of aging in Drosophila: Along the giant-fiber sensory-motor pathway. Paper presented at the Society for Neuroscience Annual Meeting, Chicago, IL.

Ruan, H., \& Wu, C. F. (2008). Social interaction-mediated lifespan extension of Drosophila $\mathrm{Cu} / \mathrm{Zn}$ superoxide dismutase mutants. Proceedings of the National Academy of Sciences of the United States of America, 105(21), 7506-7510. doi:10.1073/pnas.0711127105

Şahin, A., Held, A., Bredvik, K., Major, P., Achilli, T. M., Kerson, A. G., . . Reenan, R. (2017). Human SOD1 ALS Mutations in a Drosophila Knock-In Model Cause Severe Phenotypes and Reveal Dosage-Sensitive Gain- and Loss-of-Function Components. Genetics, 205(2), 707-723. doi:10.1534/genetics.116.190850

Simon, A. F., Liang, D. T., \& Krantz, D. E. (2006). Differential decline in behavioral performance of Drosophila melanogaster with age. Mechanisms of Ageing and Development, 127(7), 647-651. doi:10.1016/j.mad.2006.02.006

Simon, A. F., Shih, C., Mack, A., \& Benzer, S. (2003). Steroid control of longevity in Drosophila melanogaster. Science, 299(5611), 1407-1410.

Skorupa, D. A., Dervisefendic, A., Zwiener, J., \& Pletcher, S. D. (2008). Dietary composition specifies consumption, obesity, and lifespan in Drosophila melanogaster. Aging Cell, 7(4), 478-490. doi:10.1111/j.1474-9726.2008.00400.x

Sofola, O., Kerr, F., Rogers, I., Killick, R., Augustin, H., Gandy, C., . . Partridge, L. (2010). Inhibition of GSK-3 ameliorates Abeta pathology in an adult-onset Drosophila model of Alzheimer's disease. Plos Genetics, 6(9), e1001087. doi:10.1371/journal.pgen.1001087

Sokal, R. R., \& Rohlf, F. J. (1969). Biometry (4th ed.). New York: W. H. Freeman.

Sudmeier, L. J., Howard, S. P., \& Ganetzky, B. (2015). A Drosophila model to investigate the neurotoxic side effects of radiation exposure. Disease Models \& Mechanisms, 8(7), 669677.

Sujkowski, A., Bazzell, B., Carpenter, K., Arking, R., \& Wessells, R. J. (2015). Endurance exercise and selective breeding for longevity extend Drosophila healthspan by overlapping mechanisms. Aging, 7(8), 535-552. doi:10.18632/aging.100789

Tallis, R., Hall, G., Craig, I., \& Dean, A. (1991). How common are epileptic seizures in old age? Age and Ageing, 20(6), 442-448. doi:10.1093/ageing/20.6.442

Tamura, T., Chiang, A.-S., Ito, N., Liu, H.-P., Horiuchi, J., Tully, T., \& Saitoe, M. (2003). Aging specifically impairs amnesiac-dependent memory in Drosophila. Neuron, 40(5), 10031011.

Tanouye, M. A., \& Wyman, R. J. (1980). Motor outputs of giant nerve fiber in Drosophila. Journal of Neurophysiology, 44(2), 405-421. 
1094

1095

1096

1097

1098

1099

1100

1101

1102

1103

1104

1105

1106

1107

1108

1109

1110

1111

1112

1113

1114

1115

1116

1117

1118

1119

1120

1121

1122

1123

1124

1125

1126

1127

1128

1129

1130

1131

1132

1133

1134

1135

1136

1137

1138
Toivonen, J. M., \& Partridge, L. (2009). Endocrine regulation of aging and reproduction in Drosophila. Molecular and Cellular Endocrinology, 299(1), 39-50.

Tower, J. (1996). Aging mechanisms in fruit files. Bioessays, 18(10), 799-807. doi:10.1002/bies.950181006

Tower, J. (2011). Heat shock proteins and Drosophila aging. Experimental Gerontology, 46(5), 355-362. doi:10.1016/j.exger.2010.09.002

Trimarchi, J. R., \& Schneiderman, A. M. (1995a). Different neural pathways coordinate Drosophila flight initiations evoked by visual and olfactory stimuli. Journal of Experimental Biology, 198(Pt 5), 1099-1104.

Trimarchi, J. R., \& Schneiderman, A. M. (1995b). Flight initiations in Drosophila melanogaster are mediated by several distinct motor patterns. Journal of Comparative Physiology. A: Sensory, Neural, and Behavioral Physiology, 176(3), 355-364. doi:10.1007/bf00219061

Trout, W. E., \& Kaplan, W. D. (1970). A relation between longevity, metabolic rate, and activity in shaker mutants of Drosophila melanogaster. Experimental Gerontology, 5(1), 83-92. doi:10.1016/0531-5565(70)90033-1

Ueda, A., Iyengar, A., \& Wu, C.-F. (2021). Differential effects on neuromuscular physiology between Sod1 loss-of-function mutation and paraquat-induced oxidative stress in Drosophila. microPublication biology, 2021, 10.17912/micropub.biology.000385. doi:10.17912/micropub.biology.000385

Ueda, A., Woods, S., McElree, I., O’Harrow, T. C., Inman, C., Thenuwara, S., . . Iyengar, A. (2018). Two novel forms of ERG oscillation in Drosophila: age and activity dependence. Journal of Neurogenetics, 32(2), 118-126.

Vaupel, J. W., Carey, J. R., Christensen, K., Johnson, T. E., Yashin, A. I., Holm, N. V., . . Curtsinger, J. W. (1998). Biodemographic trajectories of longevity. Science, 280(5365), 855-860. doi:10.1126/science.280.5365.855

von Reyn, C. R., Nern, A., Williamson, W. R., Breads, P., Wu, M., Namiki, S., \& Card, G. M. (2017). Feature Integration Drives Probabilistic Behavior in the Drosophila Escape Response. Neuron, 94(6), 1190-1204.e1196. doi:10.1016/j.neuron.2017.05.036

Woods, S. A. (2017). Behavioral and physiological effects of oxidative stress throughout the lifecycle of Drosophila sod1 mutants. (MS). University of Iowa, Iowa City.

Wu, C.-F., \& Ruan, H. (2008). Social interaction-mediated lifespan extension and electrophysiological correlates in Drosophila $\mathrm{Cu} / \mathrm{Zn}$ superoxide dismutase mutants. Paper presented at the Society for Neuroscience Annual Meeting, Washington DC.

Yamazaki, D., Horiuchi, J., Nakagami, Y., Nagano, S., Tamura, T., \& Saitoe, M. (2007). The Drosophila DCO mutation suppresses age-related memory impairment without affecting lifespan. Nature Neuroscience, 10(4), 478-484.

Yeoman, M., \& Faragher, R. (2001). Ageing and the nervous system: insights from studies on invertebrates. Biogerontology, 2(2), 85-97.

Zhao, X. L., Wang, W. A., Tan, J. X., Huang, J. K., Zhang, X., Zhang, B. Z., . . Huang, F. D. (2010). Expression of beta-amyloid induced age-dependent presynaptic and axonal changes in Drosophila. Journal of Neuroscience, 30(4), 1512-1522. doi:10.1523/jneurosci.3699-09.2010

Ziegler, D. V., Wiley, C. D., \& Velarde, M. C. (2015). Mitochondrial effectors of cellular senescence: beyond the free radical theory of aging. Aging Cell, 14(1), 1-7. 
Table 1. Features of GF Pathway SLR and LLR in young and aged flies

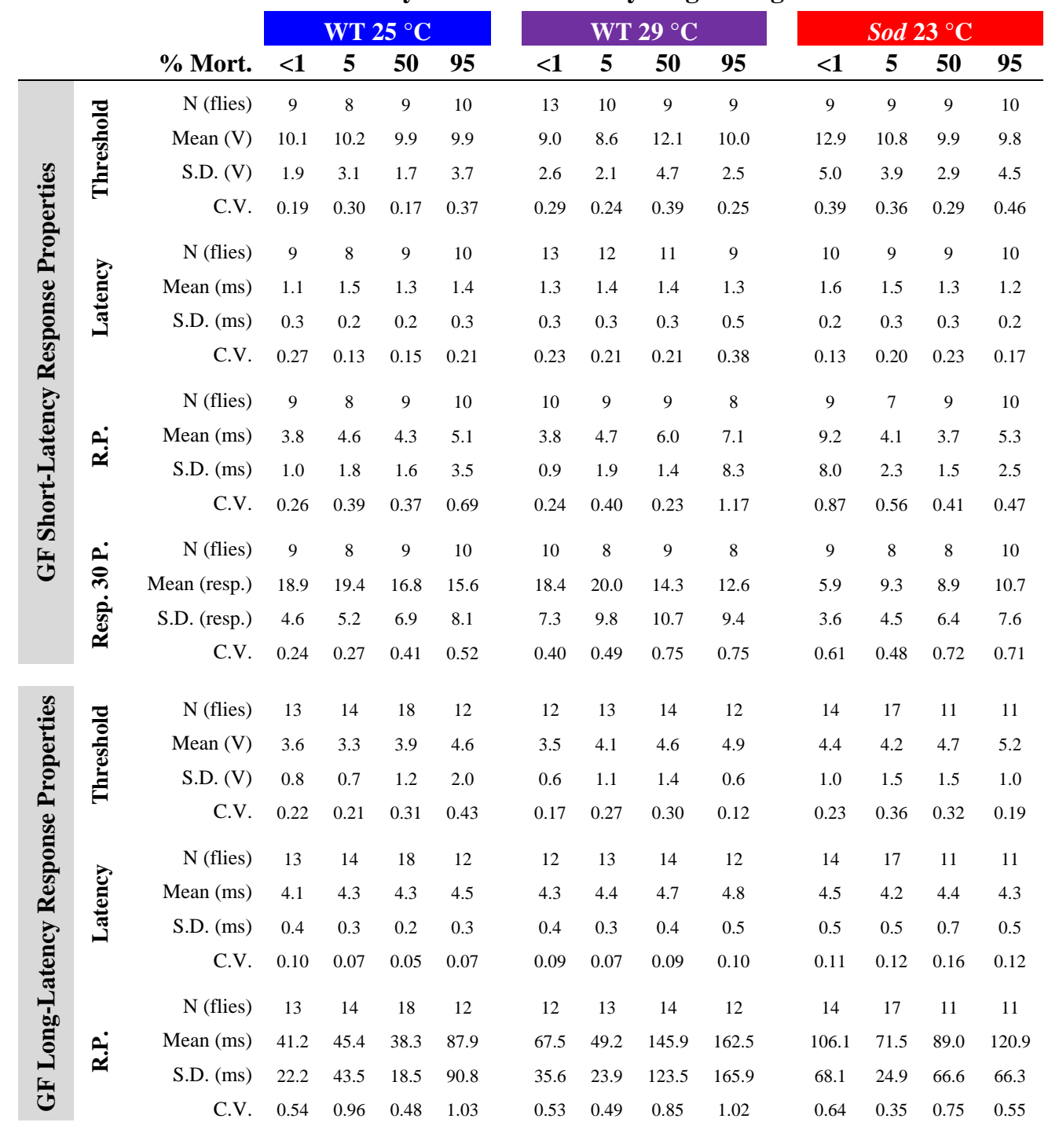

1141 

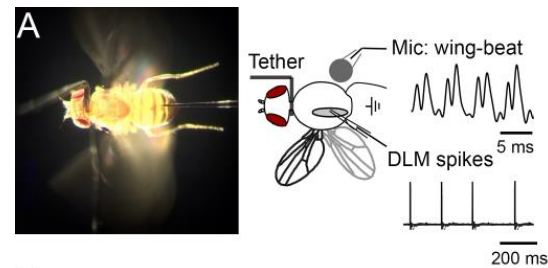

D WT $25^{\circ} \mathrm{C}-17 \mathrm{~d}$

$54 \mathrm{~d}$

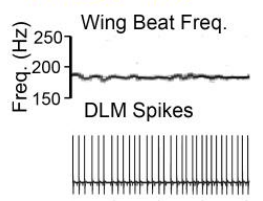

WT $29^{\circ} \mathrm{C}$ - 12 d

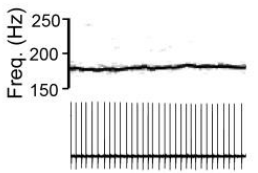

Sod $23^{\circ} \mathrm{C}-6 \mathrm{~d}$

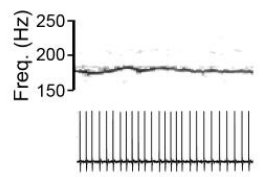

1142

1143

1144

1145

1146

1147

1148

1149

1150

1151

1152

1153

1154

1155

1156

1157

1158

1159

1160

1161

1162 $0.01, * * * \mathrm{p}<0.001$.
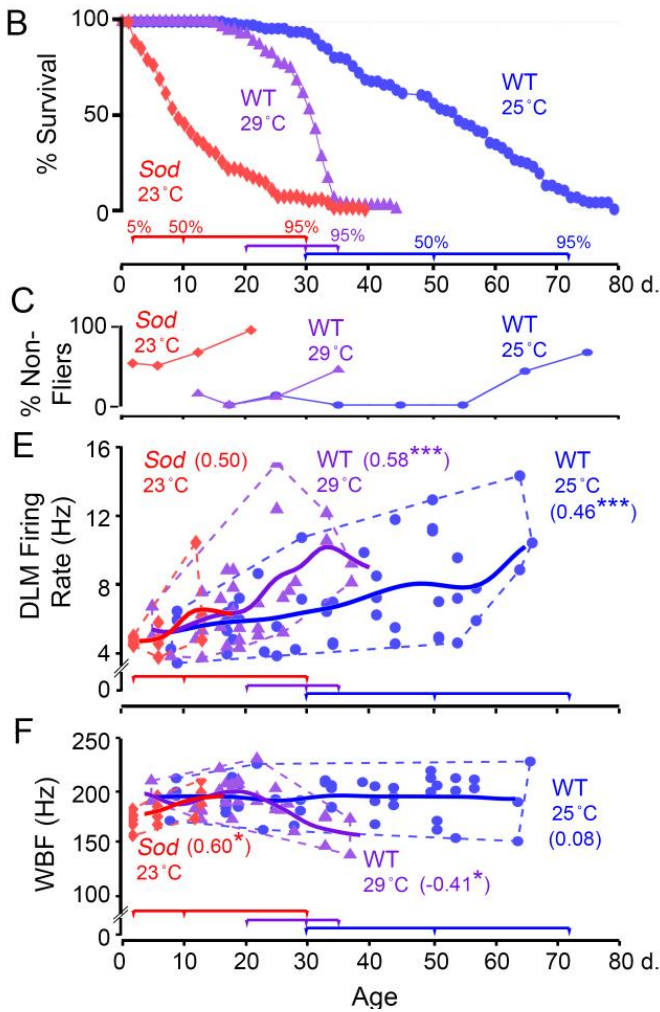

Figure 1. Flight motor performance across the lifespan of WT control and Sod mutant flies. (A, Left) Photograph of tethered fly preparation used in the flight and electrophysiological assays. Spiking activity in the DLM was monitored by a tungsten microelectrode, and acoustic signals generated by wing beats were picked up via a microphone placed below the fly. (Right) A schematic drawing of the preparation and sample waveforms of the respective signals. (B) Lifespan curves of WT flies (reared at $25^{\circ} \mathrm{C}$, blue circles, $n=110$; and $29^{\circ} \mathrm{C}$, purple triangles, $n$ $=104$ ), and $\operatorname{Sod}$ mutants (red diamonds, reared at $23{ }^{\circ} \mathrm{C}, \mathrm{n}=134$ ). Colored timelines below indicate the ages corresponding to 5\%, 50\% and 95\% mortality for the respective curves. See Extended Data Figure 1-1 for log-transformed mortality curves. (C) The proportion of non-fliers (see Methods for definition) across the lifespan ( $\mathrm{n}>10$ for each point). (D) Representative spectrograms of the microphone signal (upper panels) indicating the wing beat frequency (WBF) and corresponding traces of DLM spiking (lower panels) during sustained flight in younger (left column) and aged flies (right column) from the populations tested. (E-F) Scatterplots of the DLM firing rate (E) and WBF (F) during sustained flight in $25^{\circ} \mathrm{C}$ - and $29^{\circ} \mathrm{C}$-reared WT flies (blue circles and purple triangles respectively), and in $\operatorname{Sod}$ mutants (red diamonds). Trend lines represent a Gaussian-kernel running average of the age-trajectory for the three populations (kernel window sizes of 10, 6 and $6 \mathrm{~d}$ respectively). The age-dependent Spearman's rank correlation coefficient $\left(r_{s}\right)$ is indicated for each population $(n=48,33$, and 15 flies for WT 25 ${ }^{\circ} \mathrm{C}$-, WT $29{ }^{\circ} \mathrm{C}$-reared and Sod respectively). For this and following figures, $* \mathrm{p}<0.05$, ** $\mathrm{p}<$ 


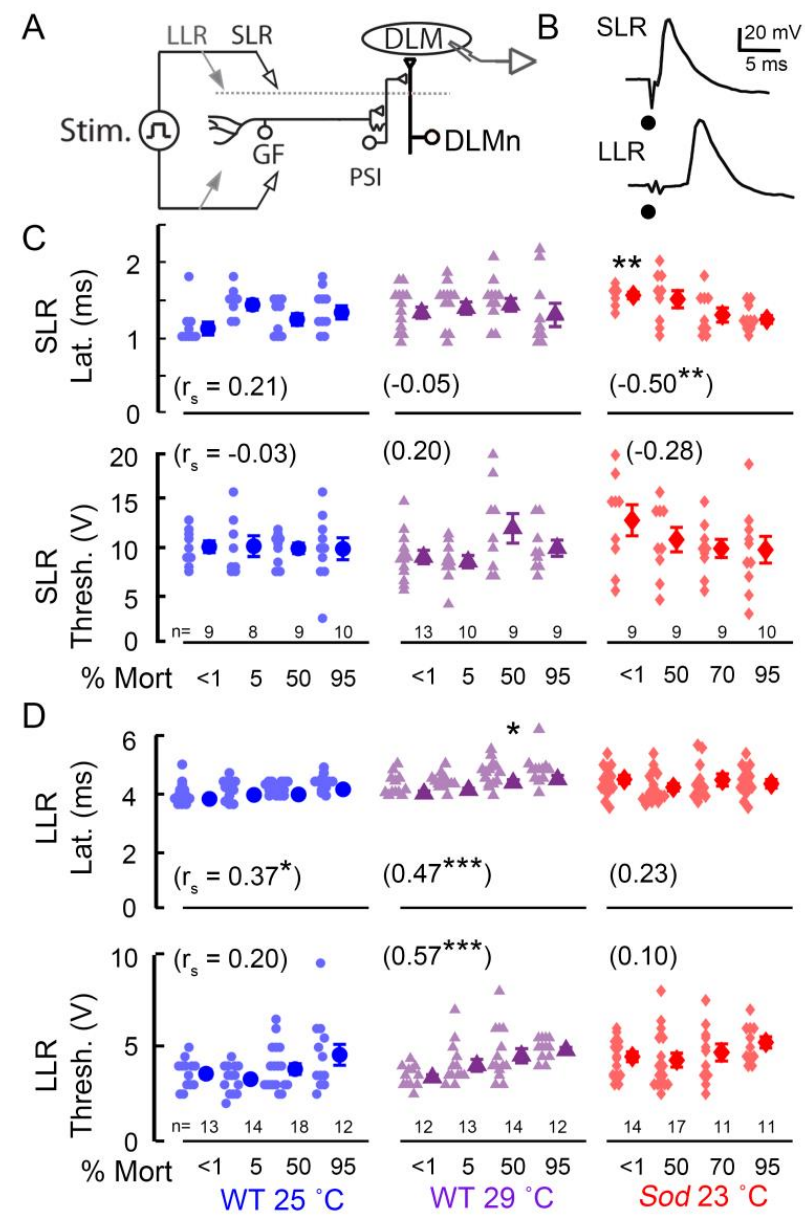

1163 Figure 2. Performance characteristics of the GF pathway and its afferent inputs in aged 1164 WT and Sod mutant flies.

1165 (A) Overview of the GF "jump-and-flight" escape pathway. The GF neuron activates the 1166 indirect flight muscle DLM through a peripherally-synapsing interneuron (PSI) which innervates 1167 the DLM motor neuron (DLMn). For clarity, the GF branch that directly innervates the jump 1168 muscle (TTM) is not shown. (B) Electrical stimulation across the brain activates the GF pathway, 1169 and motor output is monitored via DLM action potentials in the tethered fly. High-intensity stimuli directly activate the GF neuron, giving rise to short-latency responses (SLRs). Lowerintensity stimuli recruit GF afferent inputs, resulting in the DLM long-latency responses (LLRs). Dots indicate stimulus artifact. (C-D) SLR and LLR latency and threshold across the lifespan of WT and Sod flies. In this figure and in Figures 3 and 5, mean and SEM are indicated to the right of each dataset. Each lighter colored data point represents measurement from a single fly. Asterisks indicate significant difference against mortality-matched, $25^{\circ} \mathrm{C}$-reared WT flies (Kruskal-Wallis ANOVA, rank-sum post hoc test). The numbers in parenthesis indicate Spearman correlation coefficient $\left(\mathrm{r}_{\mathrm{s}}\right)$ of the parameter measurements vs. age categories. 


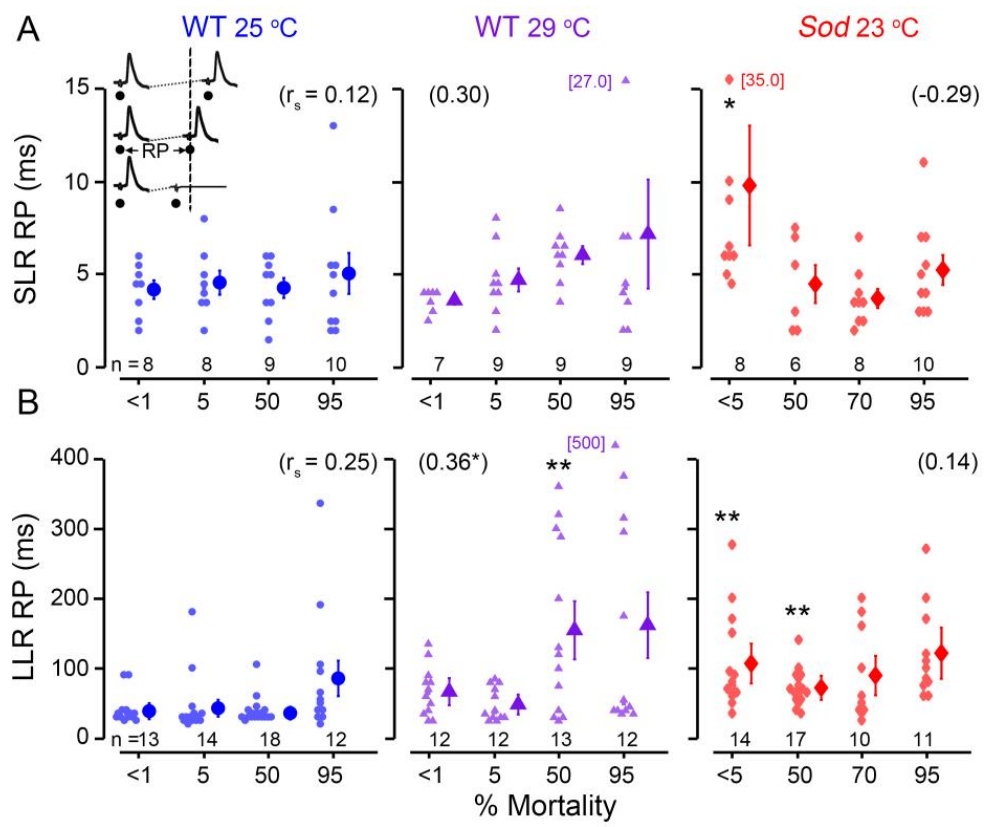

1178 Figure 3. Paired-pulse refractory characteristics of GF-mediated DLM short- and long1179 latency responses.

1180 The paired-pulse refractory period (RP) represents the shortest inter-stimulus interval where the 1181 second stimulus evokes a DLM spike; shorter stimulus intervals fail to recruit two spikes (inset). 1182 (A-B) Scatter plots of the SLR (A) and LLR (B) RPs from WT flies reared at $25^{\circ} \mathrm{C}$ and $29{ }^{\circ} \mathrm{C}$ as well as $\operatorname{Sod}$ flies reared at $23{ }^{\circ} \mathrm{C}$ are shown over the course of their lifespans, presented as percent mortality. Outlier values exceeding the axis range are indicated adjacently. See Figure 2 legend for statistical treatment and presentation. Data from the related SLR following frequency $\left(\mathrm{FF}_{30}\right)$ protocol is shown in Extended Data Figure 3-1. 

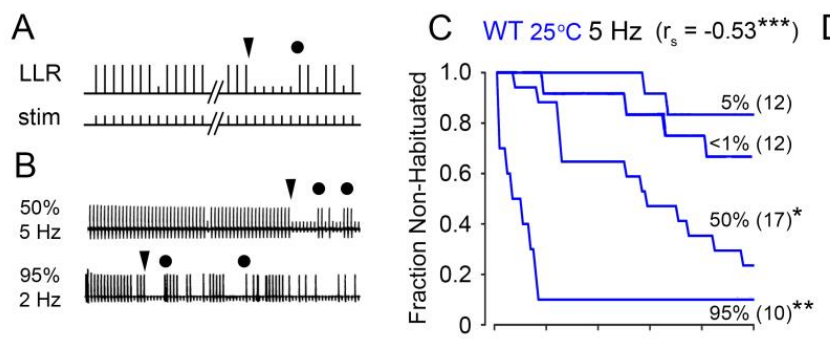

D WT $25^{\circ} \mathrm{C} 2 \mathrm{~Hz} \quad\left(-0.56^{\star \star *}\right)$
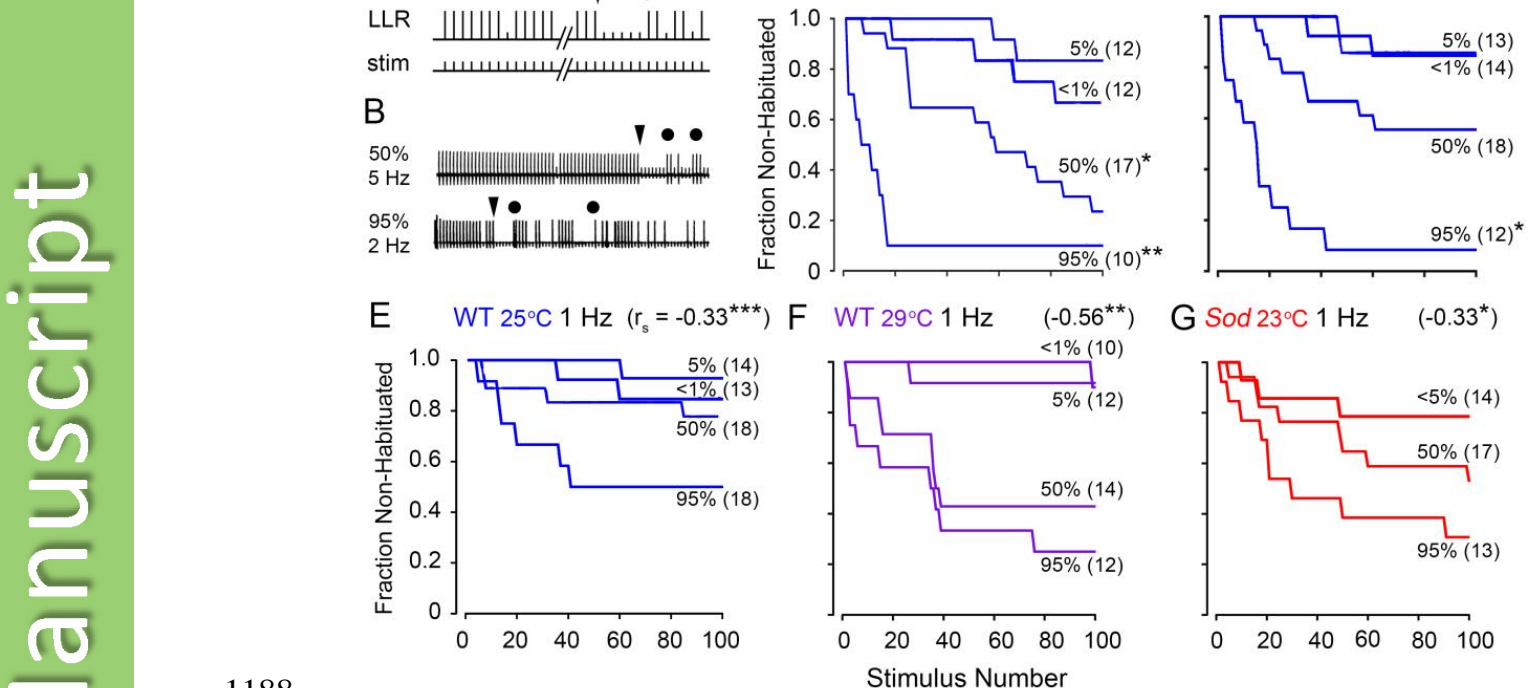

Figure 4. Age-dependent alterations in habituation kinetics of GF afferents.

(A) Habituation of GF afferents. Habituation is operationally defined as the first instant of reaching five-consecutive DLM spike failures to LLR-evoking stimulation train of defined frequency (triangle, cf. Engel \& Wu, 1996). Subsequent dis-habituation stimulus (circle, air puff) triggered recovery of LLRs, excluding sensory adaptation or motor fatigue as the basis for DLM response failure. (B) Sample traces from WT flies reared at $25{ }^{\circ} \mathrm{C}$ of slower habituation at $50 \%$ mortality (upper trace, $5 \mathrm{~Hz}$ stimulation) and faster habituation at $95 \%$ mortality (lower trace, 2 $\mathrm{Hz}$ stimulation). (C-E) Frequency dependence of LLR habituation in $25{ }^{\circ} \mathrm{C}$-reared WT flies to stimulation at $5 \mathrm{~Hz}(\mathrm{C}), 2 \mathrm{~Hz}(\mathrm{D})$, and $1 \mathrm{~Hz}(\mathrm{E})$. The fraction of flies in the sample population of each age group $(<1,5,50,95 \%$ mortality) that were not habituated after a given number of stimuli is plotted. (F-G) Habituation in WT $29^{\circ} \mathrm{C}$-reared flies (F) and in $\operatorname{Sod}$ mutants (G) to $1 \mathrm{~Hz}$ stimulation. Sample sizes as indicated next to each plot. Asterisks at the end of the trace indicate significantly faster habituation compared to habituation in mortality-matched WT $25{ }^{\circ} \mathrm{C}$-reared flies to $1 \mathrm{~Hz}$ stimulation (log-rank test). The age-dependent Spearman correlation coefficient $\left(\mathrm{r}_{\mathrm{s}}\right)$ 

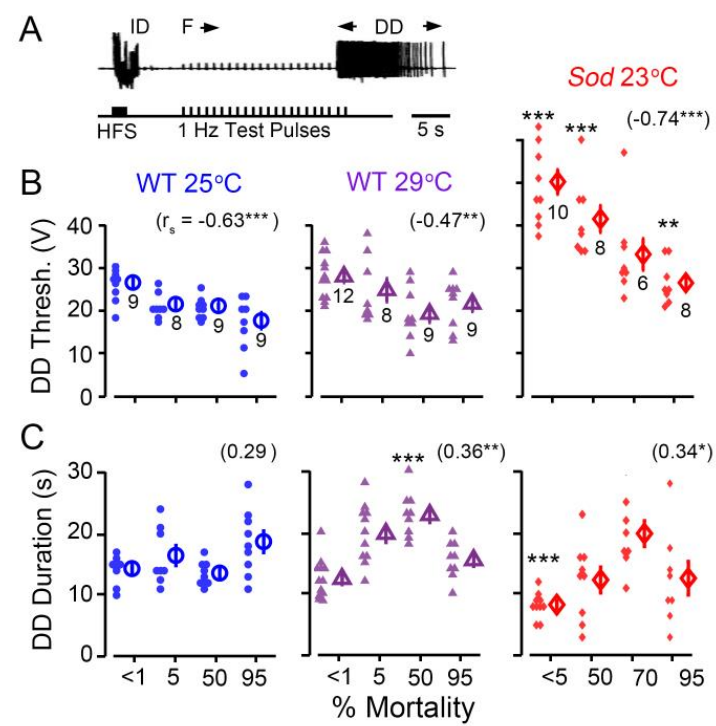

1206 Figure 5. Decreasing threshold of electroconvulsive seizure (ECS) discharges in aging flies.

1207

1208

1209

1210

1211

1212

1213

1214

1215

1216

1217
(A) ECS activity was evoked by a short train ( $2 \mathrm{~s}$ ) of high intensity, high frequency stimulation ( $0.1 \mathrm{~ms}$ pulse duration, $200 \mathrm{~Hz}$, HFS) across the head in tethered flies. DLM spike discharges serve as a convenient monitor of these seizures, and the pattern of these discharges is highly stereotypic (cf. Lee \& Wu, 2002), consisting of an initial spike discharge (ID), followed by a period of paralysis corresponding to failure of test pulse-evoked GF pathway responses (F), and a delayed spike discharge (DD). The example trace here shows the sequence in a young $25^{\circ} \mathrm{C}$ reared WT fly. (B-C) Scatterplots of the stimulation threshold to induce DD (B), and duration of the evoked DD (C) across the lifespans of $25^{\circ} \mathrm{C}$ - and $29^{\circ} \mathrm{C}$-reared WT and Sod mutants. Mean and SEM are indicated to the right of each group, sample sizes below. See Figure 2 legend for other details of statistical treatment and presentation. See Extended Data Figure 5-1 for analysis of spiking during the DD. 

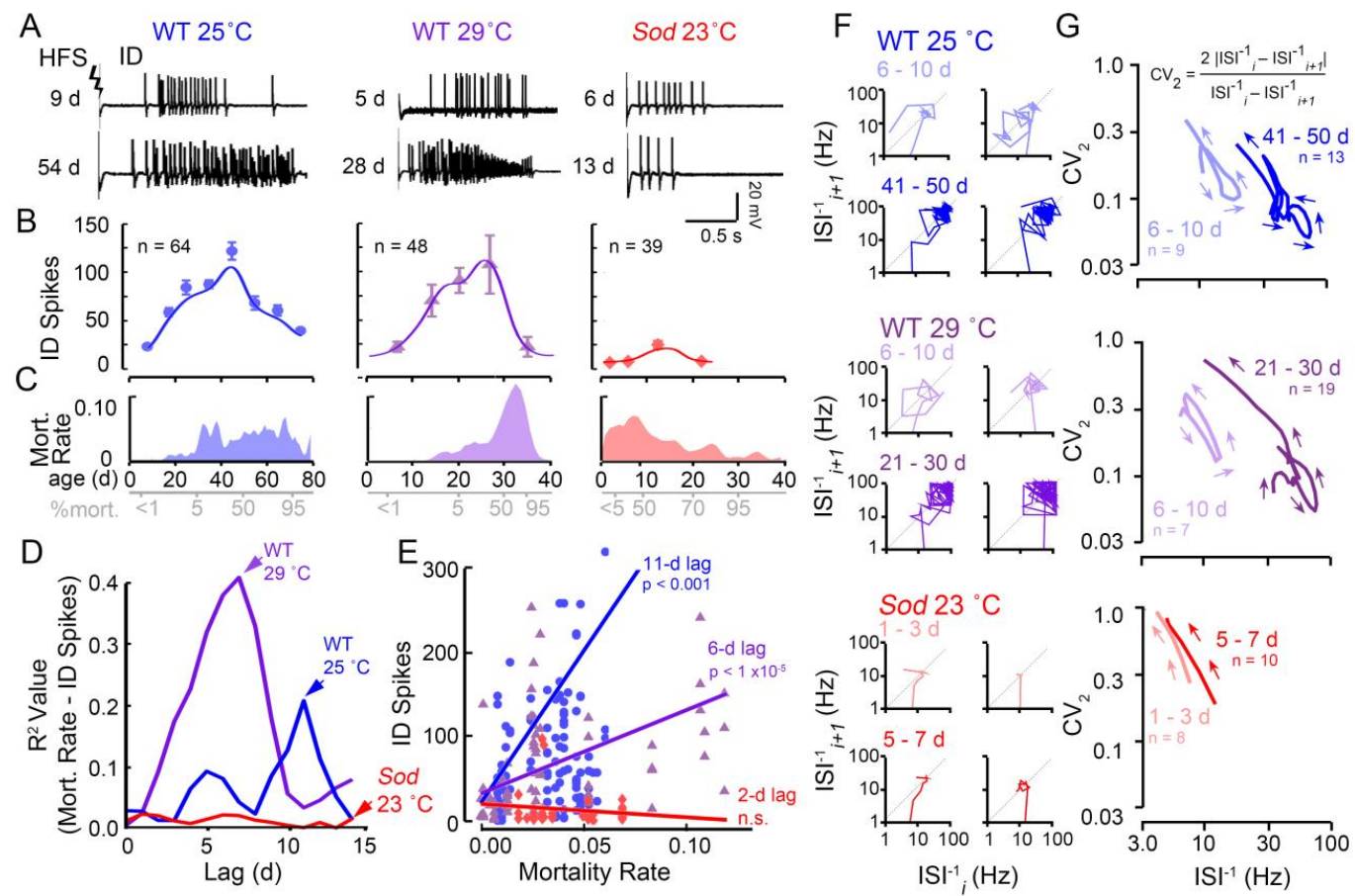

Figure 6. Age-dependent alterations in ID spike counts vs mortality rates and signatures of ID spiking trajectories in WT and Sod flies.

(A) Representative high frequency stimulation-induced IDs from young ( $<1 \%$ mortality; 9, 5 and $6 \mathrm{~d}$ traces) and aged (50\% mortality; 54, 28 and $13 \mathrm{~d}$ traces) WT and Sod flies (stimulation voltage: $80 \mathrm{~V}$ ). (B) Plot of the number of spikes within the ID across the lifespan (mean \pm SEM for different age groups, and sample sizes across the populations are indicated). Trend lines represent a Gaussian-kernel running average (see Figure 1) of the age-trajectory for the three populations. (C) Plot of the mortality rates (defined as the negative slope of the lifespan curve, Figure 1B) across the lifespan for the three populations examined (see Extended Data Figure 1-1 for log-transformed mortality rate plots). (D) Linear cross-correlation between the mortality rate and ID spike count upon introducing a lag of $0-15$ days to determine the optimal lag for maximal correlation, i.e. 11 and $6 \mathrm{~d}$ for $25^{\circ} \mathrm{C}$ and $29^{\circ} \mathrm{C}$-reared WT flies. In $\operatorname{Sod}$ mutants no significant correlation between mortality rate and ID spike count was detected. (E) Plot of best correlation between of lagged ID spike count versus mortality rate for the three populations. (pvalues as indicated). (F) Poincaré plots of representative IDs from young $(<1 \%$ mortality) and aged ( $\sim 50 \%$ mortality) flies. The instantaneous firing frequency of each inter-spike interval (ISI $\left.{ }_{i}^{1}\right)$ is plotted against the next spike interval $\left(\mathrm{ISI}^{-1}{ }_{i+1}\right.$ ) on a log scale (see text). (G) Averaged trajectories (as constructed in Lee et al., 2019) of the inter-spike interval $\left(\right.$ ISI $\left.^{-1}\right)$ versus the instantaneous coefficient of variation $\left(\mathrm{CV}_{2}\right.$, as defined by Holt et al., 1996 , see text). Lower values of $\mathrm{CV}_{2}$ indicate more rhythmic firing. Number of trajectories used to construct the average is as indicated. Note the similar changes in aged $25^{\circ} \mathrm{C}$ and $29{ }^{\circ} \mathrm{C}$-reared WT flies compared to young counterparts and distinctions between WT and Sod mutant discharges. 
A

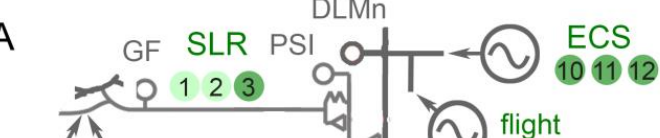
不

B
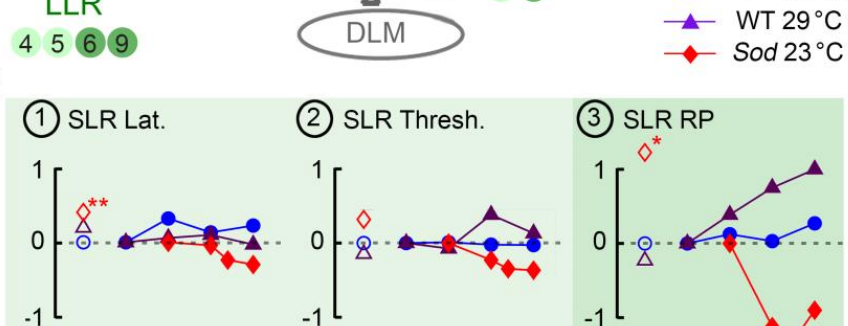

(4) LLR Lat.

(2) SLR Thresh.

(3) SLR RP

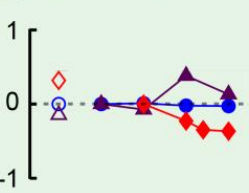

(5) LLR Thresh.

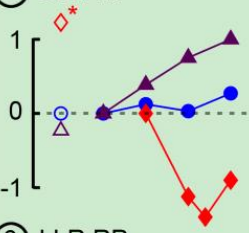

(6) LLR RP

Sod $23^{\circ} \mathrm{C}$

1242

1243

1244

1245

1246

1247

1248

1249

1250

1251

1252
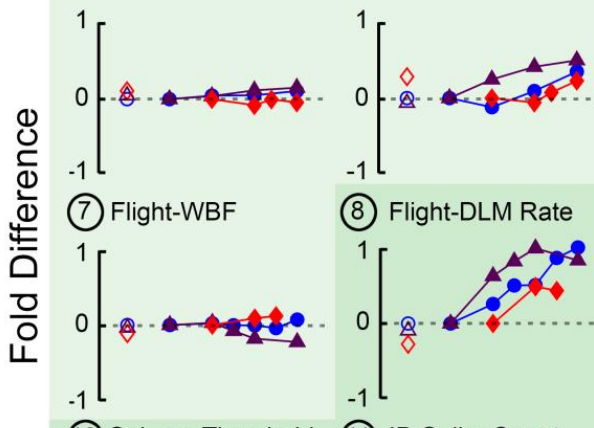

(8) Flight-DLM Rate

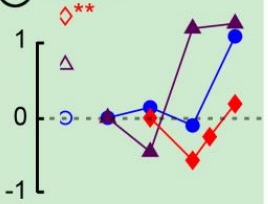

(9) LLR Habituation

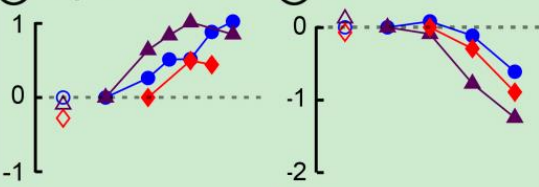

(10) Seizure Threshold

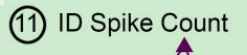

(12) DD Duration

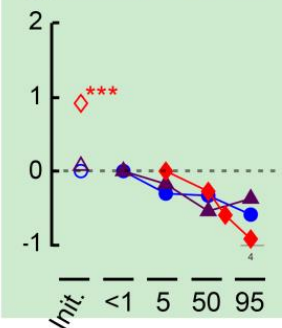

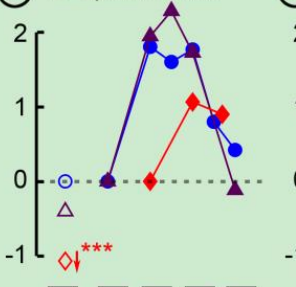

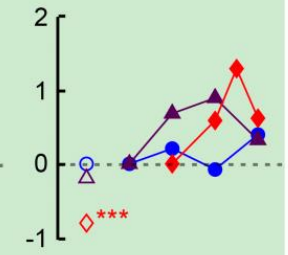

₹ $<1 \frac{\overline{5}}{50} \overline{95}$

\section{$\%$ Mortality}

Figure 7. Distinct age-trajectories of different motor circuit properties examined in this study.

(A) Schematic diagram of the circuit components generating motor patterns driving DLM activity examined in this study. Corresponding parameters displayed in panels in (B) are indicated by corresponding panel numbers. Darker backgrounds indicate that significant agedependent alterations were detected. (B) Normalized changes in neurophysiological parameters of the respective motor circuits are plotted as function of $\%$ mortality. Changes are presented in terms of 'fold change' from the initial ( $<1 \%$ mortality) value (i.e., $\log _{2}$ [value/initial value]), such that +1 indicates doubling, while -1 indicates halving of the parameter's value. First, the plot at the origin (open symbols, $<1 \%$ mortality) indicate fold changes compared to young WT $25^{\circ} \mathrm{C}$ 
1253 for WT $29{ }^{\circ} \mathrm{C}$ and $\operatorname{Sod} 23^{\circ} \mathrm{C}$ young flies. Second, the age-trajectories comparing flies of different 1254 ages to the youngest flies are indicate in fold changes (filled symbols: blue, purple and red for 1255 WT $25^{\circ} \mathrm{C}$, WT $29^{\circ} \mathrm{C}$, and $\operatorname{Sod} 23^{\circ} \mathrm{C}$ respectively). Panels representing circuit parameters that 1256 remain relatively robust across the lifespan in all three populations are shaded in lightly shaded, 1257 while parameters that display clear age-dependent alterations in at least one rearing condition are 1258 shaded darker. 

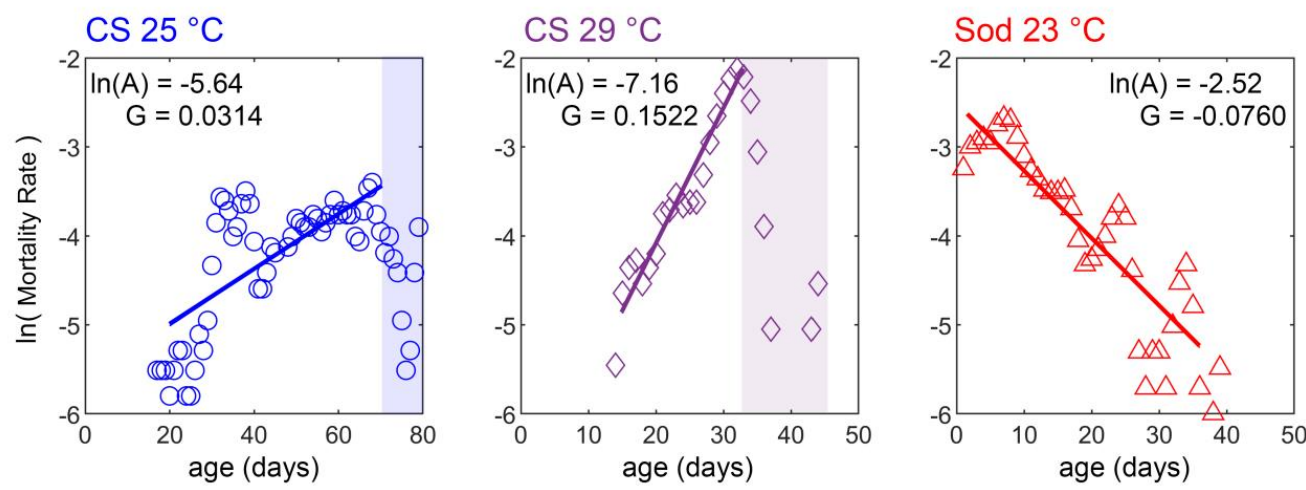

1260

1261

1262

1263

1264

1265

1266

1267

1268

1269

1270

1271

Extended Data Figure 1-1. Log-transformed mortality rate.

The mortality rate is defined as the negative slope of the survival curve (S) [i.e. mortality rate $=-1\left(\mathrm{~S}\left(t_{0}\right)-\mathrm{S}\left(t_{1}\right)\right) /\left(t_{0}-t_{1}\right)$, for the interval between time points $t_{0}$ and $\left.t_{1}\right]$. The logtransformed mortality rates corresponding to the survival curves in Figure 1B are plotted. Solid lines correspond to the linear Gompertz fit of the mortality rate distribution (i.e. $\ln$ (mortality rate) $=\ln (A)+G t)$. The fit parameters $A$ and $G$ for each distribution are indicated. For CS $25^{\circ} \mathrm{C}$ and $29^{\circ} \mathrm{C}$-reared flies, the Gompertz line is a reasonable fit for the mortality rate over most of the survival curve. However, there is a marked deviation observed in the oldest flies ( $95 \%$ survival, shaded region). The Sod lifespan curve displays a distinctive negative mortality rate slope implying a potentially distinct set of factors which contribute to mortality in these individuals. 


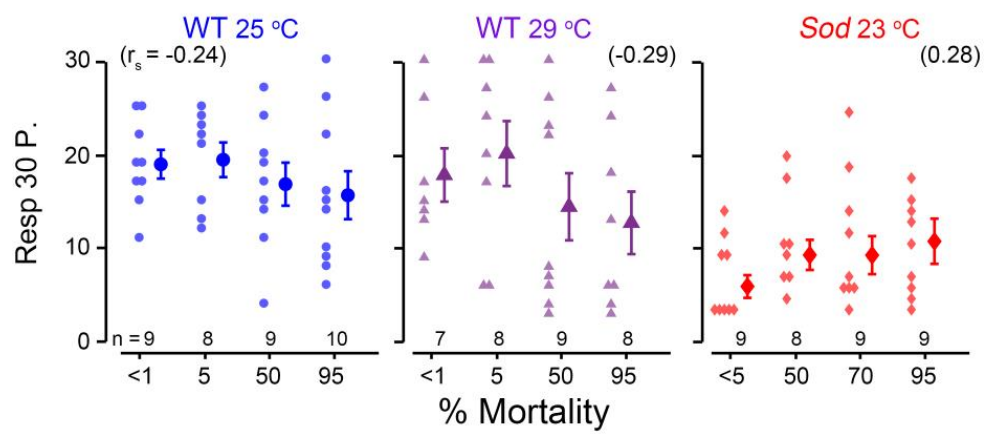

1272 Extended Data Figure 3-1. Giant-fiber SLR properties across the lifespan: $\mathbf{F F}_{\mathbf{3 0}}$ protocol.

$1273 \mathrm{The} \mathrm{FF}_{30}$ protocol measures the ability of the GF pathway to follow high-frequency stimulation.

1274 Three trains 10 stimuli are delivered at $200 \mathrm{~Hz}$ with a $10 \mathrm{~s}$ interval between trains (30 total

1275 stimuli). The number of responses was recorded (Resp 30 P.), with a higher response rate

1276 corresponding to a better ability to follow high-frequency stimulation. Sample sizes as indicated

1277 for each age group, and the Spearman rank correlation $\left(\mathrm{r}_{\mathrm{s}}\right)$ is shown above. No population

1278 displayed statistical significance age-dependent trend (i.e. $\mathrm{p}>0.05$ ). 


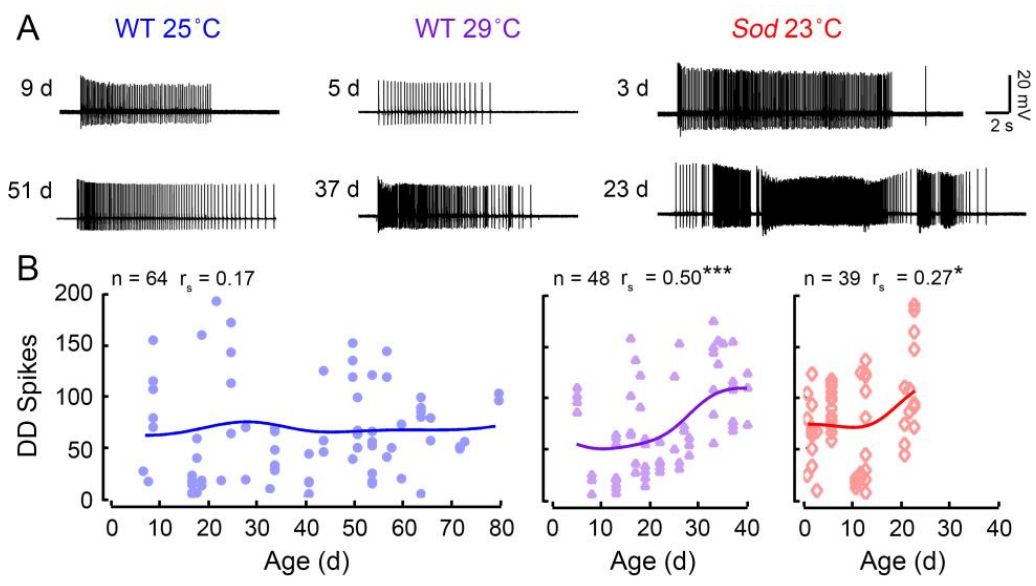

1280 Extended Data Figure 5-1. DD spiking across the lifespan. (A) Example traces of DD spiking 1281 in young (top row) and aged (bottom row) flies. (B) Scatterplot of the spike count during the DD. 1282 Trend-line indicates the Gaussian kernel running average as computed in Figure 1. The sample 1283 size and age-dependent rank correlation $\left(\mathrm{r}_{\mathrm{s}}\right)$ are indicated above. $(* \mathrm{p}<0.05, * * * \mathrm{p}<0.001)$. 